\title{
Assessment of Ambient Day Indoor and Outdoor Noise Levels in University of Science and Technology Port Harcourt
}

\author{
Tubonimi Joseph Kio Ideriah \\ Institute of Pollution Studies, Rivers State University of Science and Technology Nkpolu Oroworukwo Port Harcourt, Rivers State, Nigeria
}

Email address:

tjkideriah@ymail.com (T. J. K. Ideriah)

To cite this article:

Tubonimi Joseph Kio Ideriah. Assessment of Ambient Day Indoor and Outdoor Noise Levels in University of Science and Technology Port Harcourt. International Journal of Science, Technology and Society. Vol. 3, No. 6, 2015, pp. 265-278. doi: 10.11648/j.ijsts.20150306.11

\begin{abstract}
Indoors and outdoors ambient noise levels were measured within the RSUST to determine the levels and sources of noise pollution within the university campus in order to ascertain if the noise level has exceeded acceptable limit to affect the air quality of the environment and also access the impact of various activities in the university with regard to noise pollution. Noise levels were measured with a digital sound level meter model 407730 manufactured by EXTECH instruments with "A" weighting scale. The measured noise levels ranged between $47.8 \mathrm{~dB}(\mathrm{~A})$ and $103.2 \mathrm{~dB}$ (A). The mean noise levels recorded around the $2000 \mathrm{KVA}$ and $1275 \mathrm{KVA}$ generators exceeded the permissible noise levels guideline of $90 \mathrm{~dB}$ (A) recommended by Occupational Safety Health Agency and Federal Ministry of Environment while all the mean noise levels measured within the university exceeded the guideline values of $35-55 \mathrm{~dB}$ (A) set by World Health Organization. High positive correlation coefficients between indoor and outdoor were recorded. Analysis of variance on noise levels between hostels, road junctions, gates and Departments showed significant difference $(\mathrm{P}<0.05)$. Noise levels at outdoors were generally higher than indoors. $\mathrm{t}$ - test on the mean noise levels between indoor and outdoor were not significant $(\mathrm{P}>0.05)$ but $\mathrm{t}-$ test between maximum and minimum noise levels and between the Departments were significant $(\mathrm{P}<0.05)$. The measured noise levels were typical of urban communities in developing countries. In addition to steady electricity supply from National grid, solar plant or sound proof generators were recommended.
\end{abstract}

Keywords: Ambient, Noise, University, Road Junction, Occupational Exposure, Sound Meter, Indoor, Outdoor

\section{Introduction}

The study area University of Science and Technology is located within Port Harcourt metropolis in the Niger Delta sedimentary basin of Nigeria. Port Harcourt lies within latitudes $4^{\circ} 43^{\prime} 07^{\prime \prime}$ and $4^{\circ} 54^{\prime} 32^{\prime \prime} \mathrm{N}$ and longitudes $6^{\circ} 56^{\prime} 04^{\prime \prime}$ and $7^{\circ} 03^{\prime} 20^{\prime \prime} \mathrm{E}$ with a mean annual rainfall of over $2000 \mathrm{~mm}$ and mean annual temperature of $29^{\circ} \mathrm{C}$ (NMS, 1998). The university has staff strength of 1,850 and a student population of 15, 376 (RSUST, 2013).

There is widespread and increasing excessive noise exposure everywhere especially in developing countries. In Africa, there are high noise expose levels in the formal (manufacturing and mining) and informal occupational sector (small industries such as vehicle repairing, metal - working and milling) as well as the non - occupational sector (urban environment and leisure). Awareness of hazard amongst employers, employees and the public is however very low.
The need for studies regarding urban noise pollution and its consequences on the environment has motivated various researchers in several countries (Ugwunyi et al., 2005; Oyedepo and Saadu, 2009). Many researchers have reported that road traffic is the predominant noise source in urban areas (Bisio, 1996; Nelson, 1998). Singh and Jain (1995) reported that the measurements of noise levels in residential, industrial and commercial areas in the capital city of India, Delhi commercial areas have the highest noise levels followed by industrial and residential areas. This is contrary to the finding of Oyedepo and Saadu (2009) who carried out measurement of noise levels in busy road junctions, residential, industrial, commercial and passenger loading parks areas in Ilorin city, Nigeria. The result shows that industrial areas have the highest noise levels followed by busy roads and junctions, passengers loading parks and commercial areas. It has been generally accepted that noise pollution, particularly road traffic noise is rapidly expanding in cities such as those of South - eastern 
Nigeria (Onnu, 1992).

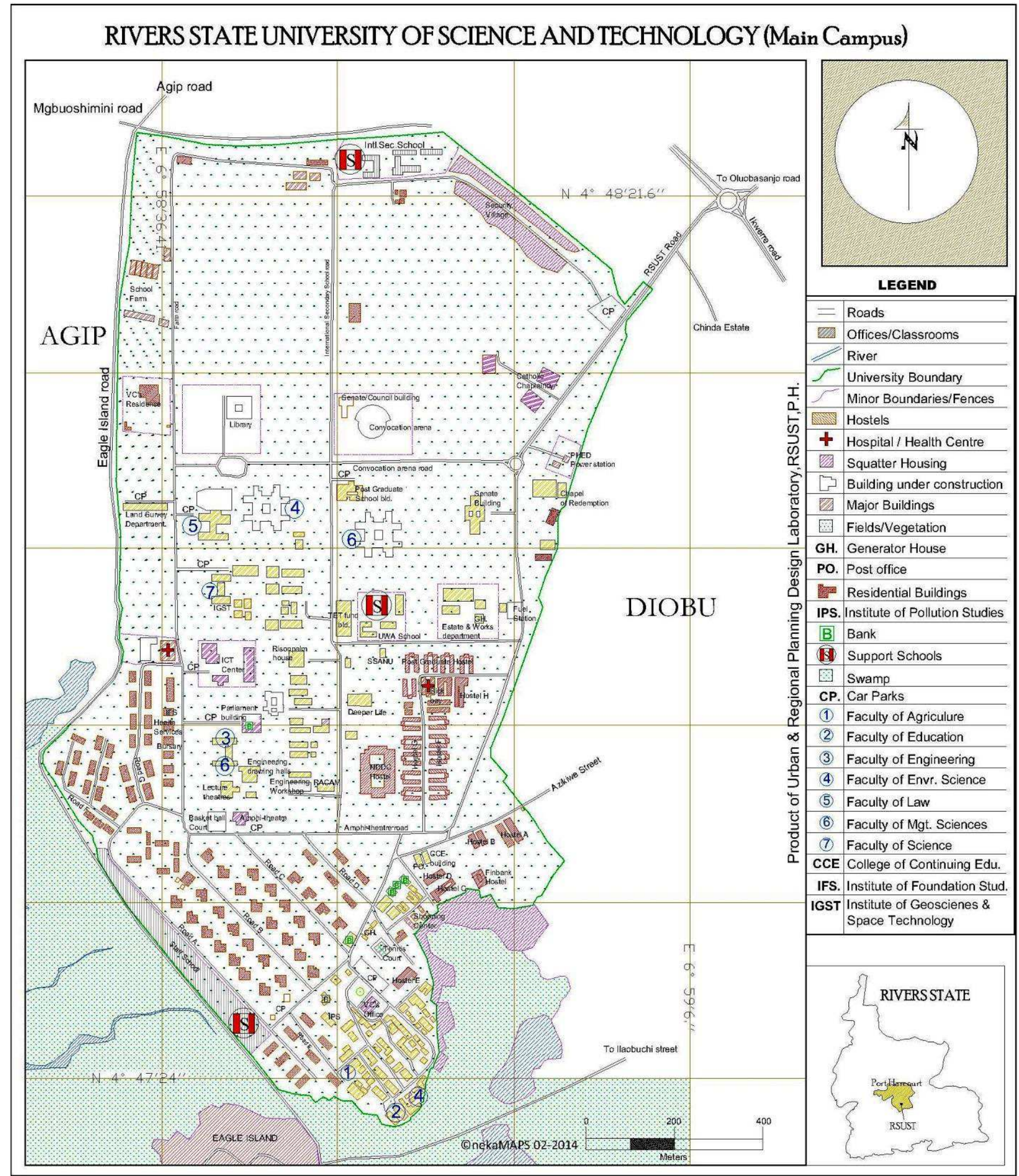

Source: Urban \& Regional Planning Dept. RSUST, P.H.

Fig. 1. Map of RSUST showing the sampling stations.

Noise does not only disturb sleep, interrupt conversation, and create stress and annoyance in the general population; it also reduces the efficiency and output of workers (Sinha and Sridharan, 1999). Noise is known to cause hearing loss, which at times may be irreversible. NIOSH (1996) attributed hearing loss among workers to their exposure in the industries while Smooreburg et al. (2003) identified prolonged equivalent daily exposures of at least $85 \mathrm{~dB}(\mathrm{~A})$ as 
a contributing factor to increased blood pressure and hypertension. Loss of hearing due to exposure to noise can equally lead to tinnitus that is buzzing in the ear while insomnia and tiring can also be caused by noise exposure. Occupational hearing loss is often being overlooked because it usually occurs insidiously without dramatic consequence such as bleeding, deformity or death (Berger, 2000).

Environmental noise is a major issue in most countries especially in heavily populated or industrialized areas. Its sources and adverse effect on humans are varied and can range from sleep disturbances to increased stress levels, potentially leading to more major problem such as heart disease in the long run.

Noise pollution affects both health and behavior. Unwanted sound (noise) can damage psychological health. Noise pollution can cause hypertension, high stress levels, tinnitus, hearing loss, sleep disturbances and other harmful effects (Rosen and Olin, 1995).

The most important factors raising noise pollution in urban areas include interalia appliances, vehicular traffic, neighbourhood electrical appliances, television and music systems, public address systems, railway and air traffic and generating sets. Even researchers fall prey to the noise generated by household equipments (Singh and Daver, 2004).

Noise in big cities is considered by the World Health Organization (WHO) to be the third most hazardous type of pollution, after air and water pollution (WHO, 2005). A study carried out by Goswami et al., (2011) on traffic noise of two university campuses in Balasore, India, found noise levels beyond permissible limit of $70 \mathrm{~dB}$ (A), during the day time. With increase in student population, staff and vehicular activities, the noise levels in RSUST campus is expected to increase. Thakur, (2006) in the study of noise around an educational institution reported that, there is need for proper land - use planning when traffic corridors are built in silence zone areas.

In most developing countries, occupational noise and urban, environmental noise (especially traffic noise) are increasing risk factors for hearing impairment. Many of these countries often lack both effective legislation against noise and programme to prevent noise - induced hearing loss (Demian et al., 2008). Where these exist, they are often poorly enforced and implemented. Most especially in Nigeria none is at high level.

Noise pollution can cause hearing impairment, both temporarily and permanently noise can cause sleepless night which may lead to students not being able to concentrate during the day. People generate noise by the use of generators, cars, machines and students making noise during and after lectures. Indoor noise can be caused by machines, building activities and music performances especially in some workplaces. Noise induced hearing loss can be caused by outside (e.g. trains) or inside (e.g. music) noise. In animals, noise can increase the risk of death by altering predator or prey detecting and avoidance, interfere with reproduction and navigator and contribute to permanent hearing loss (Jefferson, 2013).

It is therefore important to determine the level and sources of pollution within the university campus in order to ascertain if the noise level has exceeded acceptable limit. This study therefore aims to measure and obtain data and determine the major sources of noise within the university campus.

\section{Materials and Method}

\subsection{Measurement Instrument and Method}

A Cole - Parmer Extech Model 407730 sound level meter was used to measure the noise levels within the campus. The equipment has a $2 \mathrm{~dB}$ accuracy with $0.1 \mathrm{~dB}$ resolution and a measuring range of $40-130 \mathrm{~dB}$.

Table 1. Geographical Location of Measurement Sites.

\begin{tabular}{|c|c|c|c|c|}
\hline \multirow{3}{*}{$\frac{\text { Location }}{\text { Main Gate }}$} & \multicolumn{4}{|c|}{ Geographical Locations } \\
\hline & \multicolumn{2}{|c|}{ Northings (Lat.) } & \multicolumn{2}{|c|}{ Eastings (Long.) } \\
\hline & $04^{\circ} 48^{\prime}$ & $14.1^{\prime \prime}$ & $006^{\circ} 59^{\prime}$ & $07.2^{\prime \prime}$ \\
\hline Main Gate car park & $04^{\circ} 48^{\prime}$ & $12.7^{\prime \prime}$ & $006^{\circ} 59^{\prime}$ & $09.8^{\prime \prime}$ \\
\hline Azikiwe Back Gate & $04^{\circ} 47^{\prime}$ & $36.6^{\prime \prime}$ & $006^{\circ} 59^{\prime}$ & $03.0^{\prime \prime}$ \\
\hline Chemistry Lab 1 inside & $04^{\circ} 47^{\prime}$ & $52.2^{\prime \prime}$ & $006^{\circ} 58^{\prime}$ & 44. $9^{\prime \prime}$ \\
\hline Chemistry Lab 1 outside & $04^{\circ} 47^{\prime}$ & $52.3^{\prime \prime}$ & $006^{\circ} 58^{\prime}$ & $45.4^{\prime \prime}$ \\
\hline Chemistry Dept upstairs & $04^{\circ} 47^{\prime}$ & $53.0^{\prime \prime}$ & $006^{\circ} 58^{\prime}$ & 46. $4^{\prime \prime}$ \\
\hline Physics Lab 1 inside & $04^{\circ} 47^{\prime}$ & $53.3^{\prime \prime}$ & $006^{\circ} 58^{\prime}$ & 44. $7^{\prime \prime}$ \\
\hline Physics Lab 1 outside & $04^{\circ} 47^{\prime}$ & $54.3^{\prime \prime}$ & $006^{\circ} 58^{\prime}$ & 45. $6^{\prime \prime}$ \\
\hline Physics dept upstairs & $04^{\circ} 47^{\prime}$ & $54.3^{\prime \prime}$ & $006^{\circ} 58^{\prime}$ & 46. $2^{\prime \prime}$ \\
\hline Medical Lab Dept & $04^{\circ} 47^{\prime}$ & $50.5^{\prime \prime}$ & $006^{\circ} 58^{\prime}$ & 46. $2^{\prime \prime}$ \\
\hline Biology Lab 1 outside & $04^{\circ} 47^{\prime}$ & $49.5^{\prime \prime}$ & $006^{\circ} 58^{\prime}$ & $45.6^{\prime \prime}$ \\
\hline Biology Lab 1 inside & $04^{\circ} 47^{\prime}$ & $49.9^{\prime \prime}$ & $006^{\circ} 58^{\prime}$ & $45.5^{\prime \prime}$ \\
\hline Love garden & $04^{\circ} 47^{\prime}$ & $34.7^{\prime \prime}$ & $006^{\circ} 58^{\prime}$ & $56.1^{\prime \prime}$ \\
\hline UST Farm Site & $04^{\circ} 48^{\prime}$ & $09.9^{\prime \prime}$ & $006^{\circ} 58^{\prime}$ & $37.8^{\prime \prime}$ \\
\hline Hostel B (Boys) & $04^{\circ} 47^{\prime}$ & $34.7^{\prime \prime}$ & $006^{\circ} 58^{\prime}$ & $59.1^{\prime \prime}$ \\
\hline Hostel C (Girls) & $04^{\circ} 47^{\prime}$ & $29.7^{\prime \prime}$ & $006^{\circ} 58^{\prime}$ & $55.8^{\prime \prime}$ \\
\hline Outside F & $04^{\circ} 47^{\prime}$ & $41.8^{\prime \prime}$ & $006^{\circ} 58^{\prime}$ & $55.6^{\prime \prime}$ \\
\hline Front of law & $04^{\circ} 47^{\prime}$ & $57.3^{\prime \prime}$ & $006^{\circ} 58^{\prime}$ & $39.2^{\prime \prime}$ \\
\hline Business Education & $04^{\circ} 47^{\prime}$ & $17.6^{\prime \prime}$ & $006^{\circ} 58^{\prime}$ & $51.8^{\prime \prime}$ \\
\hline Urban \& Reg. Plan & $04^{\circ} 47^{\prime}$ & $15.1^{\prime \prime}$ & $006^{\circ} 58^{\prime}$ & $53.2^{\prime \prime}$ \\
\hline Estate Management & $04^{\circ} 47^{\prime}$ & $16.7^{\prime \prime}$ & $006^{\circ} 58^{\prime}$ & $55.0^{\prime \prime}$ \\
\hline Chemical/Pet. Chem Eng. & $04^{\circ} 47^{\prime}$ & $44.1^{\prime \prime}$ & $006^{\circ} 58^{\prime}$ & $46.3^{\prime \prime}$ \\
\hline Civil Engineering & $04^{\circ} 47^{\prime}$ & $42.0^{\prime \prime}$ & $006^{\circ} 58^{\prime}$ & $45.2^{\prime \prime}$ \\
\hline Electrical Engineering & $04^{\circ} 47^{\prime}$ & $40.5^{\prime \prime}$ & $006^{\circ} 58^{\prime}$ & $45.6^{\prime \prime}$ \\
\hline Mechanical Engineering & $04^{\circ} 47^{\prime}$ & $50.4^{\prime \prime}$ & $006^{\circ} 58^{\prime}$ & $50.4^{\prime \prime}$ \\
\hline $\mathrm{NEH}$ & $04^{\circ} 47^{\prime}$ & $46.2^{\prime \prime}$ & $006^{\circ} 58^{\prime}$ & $46.5^{\prime \prime}$ \\
\hline Eco bank Premises & $04^{\circ} 47^{\prime}$ & $42.3^{\prime \prime}$ & $006^{\circ} 58^{\prime}$ & $42.8^{\prime \prime}$ \\
\hline MDR Outside & $04^{\circ} 47^{\prime}$ & $39.7^{\prime \prime}$ & $006^{\circ} 58^{\prime}$ & $43.5^{\prime \prime}$ \\
\hline MDR Inside & $04^{\circ} 47^{\prime}$ & $38.7^{\prime \prime}$ & $006^{\circ} 58^{\prime}$ & $41.3^{\prime \prime}$ \\
\hline EN 10 Outside & $04^{\circ} 47^{\prime}$ & $40.7^{\prime \prime}$ & $006^{\circ} 58^{\prime}$ & $40.1^{\prime \prime}$ \\
\hline EN 10 Inside & $04^{\circ} 47^{\prime}$ & $41.9^{\prime \prime}$ & $006^{\circ} 58^{\prime}$ & $40.8^{\prime \prime}$ \\
\hline ISS Gate & $04^{\circ} 48^{\prime}$ & $22.4^{\prime \prime}$ & $006^{\circ} 58^{\prime}$ & $50.1^{\prime \prime}$ \\
\hline ISS senior block & $04^{\circ} 48^{\prime}$ & $21.5^{\prime \prime}$ & $006^{\circ} 58^{\prime}$ & $55.4^{\prime \prime}$ \\
\hline ISS field & $04^{\circ} 48^{\prime}$ & $23.6^{\prime \prime}$ & $006^{\circ} 58^{\prime}$ & $51.2^{\prime \prime}$ \\
\hline Library & $04^{\circ} 48^{\prime}$ & $02.6^{\prime \prime}$ & $006^{\circ} 58^{\prime}$ & $41.1^{\prime \prime}$ \\
\hline Security village Power Min. & $04^{\circ} 48^{\prime}$ & $22.5^{\prime \prime}$ & $006^{\circ} 58^{\prime}$ & $51.3^{\prime \prime}$ \\
\hline Security village Deeper life & $04^{\circ} 48^{\prime}$ & $20.9^{\prime \prime}$ & $006^{\circ} 59^{\prime}$ & $02.1^{\prime \prime}$ \\
\hline Security village by car park & $04^{\circ} 48^{\prime}$ & $17.5^{\prime \prime}$ & $006^{\circ} 59^{\prime}$ & $07.0^{\prime \prime}$ \\
\hline
\end{tabular}

The equipment measures noise via a microphone probe that generates signal approximately proportional to the located sound waves. The meter has a weighting of " $\mathrm{A}$ " and "C" and "slow" and "fast" modes. The measurements were made using the slow mode and " $\mathrm{A}$ " weighting. With " $\mathrm{A}$ " 
weighting the meter responds as human ear. It is used for environmental measurements, Occupational Safety Health Agency (OSHA) regulatory testing, law enforcement and work place. Measurements were made outdoors. The meter was hand held at a height of 1.5 meters above ground and $3 \mathrm{~m}$ away from buildings or other sound reflecting sources. Geographical positioning system (GPS) was used to determine the positions of the selected areas.

\subsection{Measurement Locations/Duration/Frequency}

This study will cover Hostels B, F \& G (boys); Hostel C (girls), lecture halls of Faculties of Engineering, Science, Technical and Science, Environmental Management and Law. Also measurements will be made along major roads junctions in the campus, Farm Site and Library, International secondary school (ISS) and Security village. The noise levels (indoor and outdoor) at the selected areas were monitored daily within the hours of 8 am to $5 \mathrm{pm}$ for 2 months.

\section{Results and Discussion}

\subsection{Results}

The results of noise levels measured in Rivers State University of Science and Technology are presented in
Tables $3.1-3.7$ and Figs $3.1-3.8$. The ANOVA results and correlation matrices are shown in Tables 3.8-3.10.

Table 3.1 and Fig 3.1 show data obtained around the major university gates and car park. The results ranged from $73.1-$ $85.4 \mathrm{~dB}(\mathrm{~A})$ with a maximum mean of $80.2 \mathrm{~dB}(\mathrm{~A})$ at the main gate, $70-83.0 \mathrm{~dB}(\mathrm{~A})$ with a maximum mean of 77.4 $\mathrm{dB}(\mathrm{A})$ at the main car park and $70.8-85.0 \mathrm{~dB}(\mathrm{~A})$ with a maximum mean of $78.3 \mathrm{~dB}(\mathrm{~A})$ at Azikiwe back gate.

Table 3.2 and Fig. 3.2 show the results obtained at various major road junctions. The noise levels ranged from 57.9 $79.9 \mathrm{~dB}(\mathrm{~A})$ at Chapel of Redemption roundabout, 57.0 $81.4 \mathrm{~dB}(\mathrm{~A})$ at Love Garden, $78.5-79.1 \mathrm{~dB}$ (A) at Fidelity Bank, $61.5-79.7 \mathrm{~dB}(\mathrm{~A})$ at ITC, $57.5-78.4 \mathrm{~dB}$ (A) at Library roundabout, $51.9-68.3 \mathrm{~dB}(\mathrm{~A})$ at Vice Chancellor's Lodge, $62.0-73.4 \mathrm{~dB}(\mathrm{~A})$ at $\mathrm{PG} /$ Arena, $61.8-78.0 \mathrm{~dB}(\mathrm{~A})$ at TFC Building and $52.2-81.1 \mathrm{~dB}(\mathrm{~A})$ at RACAM with a maximum mean of $78.8 \mathrm{~dB}(\mathrm{~A})$.

Table 3.3 and Fig. 3.3 show the mean indoor and outdoor noise levels at various Departments in the University. The indoor noise levels ranged from $50.4 \mathrm{~dB}$ (A) (Law) to 83.0 $\mathrm{dB}$ (A) (Physics) with a maximum mean of $81.3 \mathrm{~dB}$ (A) (Physics). Outdoor noise levels ranged from $54.4 \mathrm{~dB}$ (A) (Business Education) to $85.8 \mathrm{~dB}$ (A) (Hostel F) with a maximum mean of $81.0 \mathrm{~dB}(\mathrm{~A})$ (Hostel C).

Table 3.1. Ambient Noise Levels at University Gates.

\begin{tabular}{|c|c|c|c|c|c|}
\hline \multirow{2}{*}{ Location } & \multirow{2}{*}{ Date } & \multirow{2}{*}{ Time } & \multicolumn{3}{|c|}{ Noise Levels dB (A) } \\
\hline & & & Maximum & Minimum & Mean \\
\hline \multirow{7}{*}{ Main Gate } & $02 / 04 / 15$ & $07: 55 \mathrm{am}$ & 81.5 & 74.6 & 78.1 \\
\hline & $03 / 04 / 15$ & 11: $44 \mathrm{am}$ & 82.6 & 75.2 & 78.9 \\
\hline & $07 / 04 / 15$ & 10: $22 \mathrm{am}$ & 84.4 & 73.1 & 78.8 \\
\hline & $08 / 04 / 15$ & $02: 23 \mathrm{pm}$ & 85.4 & 75.0 & 80.2 \\
\hline & $10 / 04 / 15$ & 02: $03 \mathrm{pm}$ & 83.3 & 74.8 & 79.1 \\
\hline & $28 / 04 / 15$ & 02: $19 \mathrm{pm}$ & 83.3 & 74.8 & 79.1 \\
\hline & $01 / 05 / 15$ & $03: 38 \mathrm{pm}$ & 82.8 & 75.2 & 79.0 \\
\hline \multirow{6}{*}{ Main Gate Car park } & $02 / 04 / 15$ & 08: 00am & 81.7 & 70.4 & 76.1 \\
\hline & $03 / 04 / 15$ & 11: $39 \mathrm{am}$ & 82.9 & 71.8 & 77.4 \\
\hline & $07 / 04 / 15$ & 11: $51 \mathrm{am}$ & 81.6 & 70.4 & 76.0 \\
\hline & $08 / 04 / 15$ & $01: 56 \mathrm{pm}$ & 81.8 & 70.7 & 76.3 \\
\hline & $10 / 04 / 15$ & $02: 28 \mathrm{pm}$ & 83.0 & 71.2 & 77.1 \\
\hline & $28 / 04 / 15$ & $02: 29 \mathrm{pm}$ & 80.8 & 70.7 & 75.8 \\
\hline \multirow{6}{*}{ Azikiwe Back Gate } & $08 / 04 / 15$ & $08: 35 \mathrm{am}$ & 84.5 & 70.9 & 77.7 \\
\hline & $09 / 04 / 15$ & 10: $52 \mathrm{am}$ & 84.5 & 71.0 & 77.8 \\
\hline & $10 / 04 / 15$ & $01: 11 \mathrm{pm}$ & 83.8 & 71.0 & 77.4 \\
\hline & $28 / 04 / 15$ & 02: $09 \mathrm{pm}$ & 85.0 & 71.6 & 78.3 \\
\hline & $30 / 04 / 15$ & $02: 30 \mathrm{pm}$ & 84.8 & 70.8 & 77.8 \\
\hline & $05 / 05 / 15$ & 02: $44 \mathrm{pm}$ & 84.7 & 71.2 & 78.0 \\
\hline
\end{tabular}

Table 3.2. Ambient Noise Levels at Major Road Junctions in University of Science and Technology.

\begin{tabular}{|c|c|c|c|c|c|}
\hline \multirow{2}{*}{ Location } & \multirow{2}{*}{ Date } & \multirow{2}{*}{ Time } & \multicolumn{3}{|c|}{ Noise Levels dB (A) } \\
\hline & & & Maximum & Minimum & Mean \\
\hline \multirow[t]{5}{*}{$\mathrm{C} /$ Redemption Roundabout } & $07 / 04 / 15$ & 08: 08am & 78.8 & 57.9 & 68.4 \\
\hline & $10 / 04 / 15$ & 11: $54 \mathrm{am}$ & 79.4 & 58.6 & 69.0 \\
\hline & $28 / 04 / 15$ & 11: $58 \mathrm{am}$ & 78.2 & 58.2 & 68.2 \\
\hline & $30 / 04 / 15$ & $01: 46 \mathrm{pm}$ & 79.9 & 59.6 & 69.8 \\
\hline & $01 / 05 / 15$ & 03: $48 \mathrm{pm}$ & 79.6 & 58.2 & 68.9 \\
\hline \multirow[t]{2}{*}{ Love Garden } & $01 / 04 / 15$ & 08: $26 \mathrm{am}$ & 78.5 & 57.0 & 67.8 \\
\hline & $03 / 04 / 15$ & $12: 05 \mathrm{pm}$ & 78.8 & 57.2 & 68.0 \\
\hline
\end{tabular}




\begin{tabular}{|c|c|c|c|c|c|}
\hline \multirow{2}{*}{ Location } & \multirow{2}{*}{ Date } & \multirow{2}{*}{ Time } & \multicolumn{3}{|c|}{ Noise Levels dB (A) } \\
\hline & & & Maximum & Minimum & Mean \\
\hline & & 12: $10 \mathrm{pm}$ & 78.2 & 57.6 & 67.9 \\
\hline & $07 / 04 / 15$ & $12: 21 \mathrm{pm}$ & 78.0 & 57.5 & 67.8 \\
\hline & $08 / 04 / 15$ & 01: $04 \mathrm{pm}$ & 78.0 & 57.2 & 67.6 \\
\hline & $09 / 04 / 15$ & $01: 10 \mathrm{pm}$ & 78.0 & 57.2 & 67.6 \\
\hline & $28 / 04 / 15$ & $01: 20 \mathrm{pm}$ & 81.4 & 64.7 & 73.1 \\
\hline & $29 / 04 / 15$ & $02: 51 \mathrm{pm}$ & 78.1 & 57.6 & 67.9 \\
\hline & $30 / 04 / 15$ & 03: $04 \mathrm{pm}$ & 77.9 & 57.1 & 67.5 \\
\hline & $05 / 05 / 15$ & $03: 33 \mathrm{pm}$ & 78.4 & 57.8 & 68.1 \\
\hline Fidelity Bank & $07 / 04 / 15$ & $12: 28$ & 79.1 & 78.5 & 78.8 \\
\hline \multirow[t]{3}{*}{ Info Tech Centre } & $28 / 04 / 15$ & 07: 46am & 79.7 & 61.5 & 70.6 \\
\hline & $01 / 05 / 15$ & 08: $34 \mathrm{am}$ & 76.2 & 62.0 & 69.1 \\
\hline & $04 / 05 / 15$ & 11: 49am & 76.5 & 62.2 & 69.4 \\
\hline \multirow[t]{3}{*}{ Library Roundabout } & $07 / 04 / 15$ & $12: 13 \mathrm{pm}$ & 78.4 & 57.8 & 68.1 \\
\hline & $10 / 04 / 15$ & $01: 22 \mathrm{pm}$ & 77.9 & 57.7 & 67.8 \\
\hline & $01 / 05 / 15$ & $04: 07 \mathrm{pm}$ & 77.6 & 57.5 & 67.6 \\
\hline VC Lodge & $02 / 04 / 15$ & 03: $00 \mathrm{pm}$ & 68.3 & 51.9 & 60.1 \\
\hline \multirow[t]{7}{*}{ P.G Sch. / Arena } & $03 / 04 / 15$ & 08: $20 \mathrm{am}$ & 73.0 & 62.3 & 67.7 \\
\hline & $10 / 04 / 15$ & 10: $30 \mathrm{am}$ & 72.3 & 62.5 & 67.4 \\
\hline & & 12: $00 \mathrm{pm}$ & 72.6 & 62.1 & 67.4 \\
\hline & $28 / 04 / 15$ & 01: $30 \mathrm{pm}$ & 73.2 & 63.0 & 68.1 \\
\hline & $30 / 04 / 15$ & $02: 50 \mathrm{pm}$ & 72.2 & 62.2 & 67.2 \\
\hline & & 03: $00 \mathrm{pm}$ & 73.4 & 62.0 & 67.7 \\
\hline & & 04: $00 \mathrm{pm}$ & 72.1 & 62.1 & 67.1 \\
\hline \multirow[t]{9}{*}{ TFC Building } & $01 / 04 / 15$ & 09: 18am & 77.7 & 62.1 & 69.9 \\
\hline & $07 / 04 / 15$ & 11: 04am & 77.2 & 62.5 & 69.9 \\
\hline & $10 / 04 / 15$ & 10: $20 \mathrm{am}$ & 77.5 & 62.7 & 70.1 \\
\hline & & $10: 25 \mathrm{am}$ & 77.3 & 62.3 & 69.8 \\
\hline & $28 / 04 / 15$ & 10: 49am & 77.2 & 62.5 & 69.9 \\
\hline & $29 / 04 / 15$ & $01.42 \mathrm{pm}$ & 77.6 & 61.8 & 69.7 \\
\hline & & $02: 20 \mathrm{pm}$ & 76.9 & 62.0 & 69.5 \\
\hline & & 03: $02 \mathrm{pm}$ & 77.2 & 62.3 & 69.8 \\
\hline & $05 / 05 / 15$ & 03: $08 \mathrm{pm}$ & 78.0 & 62.9 & 70.5 \\
\hline \multirow[t]{8}{*}{ RACAM } & $03 / 04 / 15$ & 10: $17 \mathrm{am}$ & 80.0 & 52.7 & 66.4 \\
\hline & & 12: $00 \mathrm{am}$ & 79.3 & 52.6 & 66.0 \\
\hline & $08 / 04 / 15$ & 01: $00 \mathrm{pm}$ & 79.0 & 52.2 & 65.6 \\
\hline & $09 / 04 / 15$ & 01: $30 \mathrm{pm}$ & 81.1 & 59.3 & 70.2 \\
\hline & $28 / 04 / 15$ & $02: 00 \mathrm{pm}$ & 79.4 & 52.5 & 66.0 \\
\hline & & $02: 30 \mathrm{pm}$ & 79.6 & 52.6 & 66.1 \\
\hline & & 03: $30 \mathrm{pm}$ & 79.8 & 52.7 & 66.3 \\
\hline & $04 / 05 / 15$ & $05: 00 \mathrm{pm}$ & 79.6 & 52.5 & 66.1 \\
\hline
\end{tabular}

Table 3.3. Indoor and Outdoor Mean Noise Levels in Various Departments in the University of Science and Technology.

\begin{tabular}{|c|c|c|c|c|}
\hline \multirow{2}{*}{ Department/Location } & \multirow{2}{*}{ Source } & \multicolumn{2}{|c|}{ Noise Levels dB (A) } & \multirow{2}{*}{ Mean } \\
\hline & & Maximum & Minimum & \\
\hline \multirow{2}{*}{ Chemistry Lab. I } & Indoor & 80.3 & 69.2 & 74.8 \\
\hline & Outdoor & 78.4 & 75.9 & 77.2 \\
\hline \multirow{2}{*}{ Physics Lab. I } & Indoor & 83.1 & 79.4 & 81.3 \\
\hline & Outdoor & 78.6 & 68.4 & 73.5 \\
\hline \multirow{2}{*}{ Biology Lab. I } & Indoor & 73.2 & 70.4 & 71.8 \\
\hline & Outdoor & 75.9 & 64.1 & 70.0 \\
\hline \multirow{2}{*}{ SC 10} & Indoor & 78.0 & 63.3 & 70.7 \\
\hline & Outdoor & 79.6 & 71.2 & 75.4 \\
\hline \multirow{2}{*}{$\mathrm{SC} 7$} & Indoor & 79.6 & 64.8 & 75.4 \\
\hline & Outdoor & 77.6 & 65.5 & 71.6 \\
\hline \multirow{4}{*}{ Hostels } & B (Boys) & 79.6 & 69.8 & 74.7 \\
\hline & $\mathrm{C}$ (Girls) & 82.8 & 79.1 & 81.0 \\
\hline & F (Outdoor) & 85.8 & 62.8 & 74.3 \\
\hline & $\mathrm{G}$ (Outdoor) & 84.4 & 68.1 & 76.3 \\
\hline \multirow{2}{*}{ Law } & Indoor $(\operatorname{LC} 4,5,7)$ & 67.0 & 50.4 & 58.7 \\
\hline & Outdoor & 80.5 & 62.3 & 71.4 \\
\hline \multirow{2}{*}{ Library } & Indoor & 78.4 & 59.8 & 69.1 \\
\hline & Outdoor & 79.1 & 63.8 & 71.5 \\
\hline \multirow{2}{*}{ Chem./Pet. Chem. Eng. } & Indoor & 80.1 & 58.2 & 69.2 \\
\hline & Outdoor & 61.9 & 58.1 & 60.0 \\
\hline Civil Eng & Indoor & 71.4 & 53.6 & 62.5 \\
\hline
\end{tabular}




\begin{tabular}{|c|c|c|c|c|}
\hline \multirow{3}{*}{ Department/Location } & \multirow{2}{*}{ Source } & \multicolumn{2}{|c|}{ Noise Levels dB (A) } & \multirow{2}{*}{ Mean } \\
\hline & & Maximum & Minimum & \\
\hline & Outdoor & 78.0 & 55.3 & 66.7 \\
\hline \multirow[t]{2}{*}{ Electrical Eng } & Indoor & 71.0 & 57.3 & 64.2 \\
\hline & Outdoor & 79.3 & 62.9 & 71.1 \\
\hline \multirow[t]{2}{*}{ Mechanical Eng } & Indoor & 75.0 & 61.4 & 68.2 \\
\hline & Outdoor & 59.9 & 55.2 & 57.6 \\
\hline \multirow[t]{2}{*}{ EN 10} & Indoor & 81.1 & 72.4 & 76.8 \\
\hline & Outdoor & 79.8 & 71.8 & 75.8 \\
\hline \multirow[t]{2}{*}{ MDR } & Indoor & 60.6 & 58.8 & 59.7 \\
\hline & Outdoor & 74.8 & 61.2 & 68.0 \\
\hline \multirow[t]{2}{*}{ Business Education } & Indoor & 80.4 & 62.4 & 71.4 \\
\hline & Outdoor & 74.2 & 54.4 & 64.3 \\
\hline \multirow[t]{2}{*}{ Urban \& Reg. Planning } & Indoor & 77.0 & 53.8 & 65.4 \\
\hline & Outdoor & 81.7 & 58.6 & 70.2 \\
\hline \multirow[t]{2}{*}{ Estate Management } & Indoor & 76.3 & 55.2 & 65.8 \\
\hline & Outdoor & 80.7 & 58.3 & 69.5 \\
\hline \multirow[t]{2}{*}{ Tech. \& Science } & Indoor & 71.1 & 53.8 & 62.5 \\
\hline & Outdoor & 75.8 & 55.2 & 65.5 \\
\hline
\end{tabular}

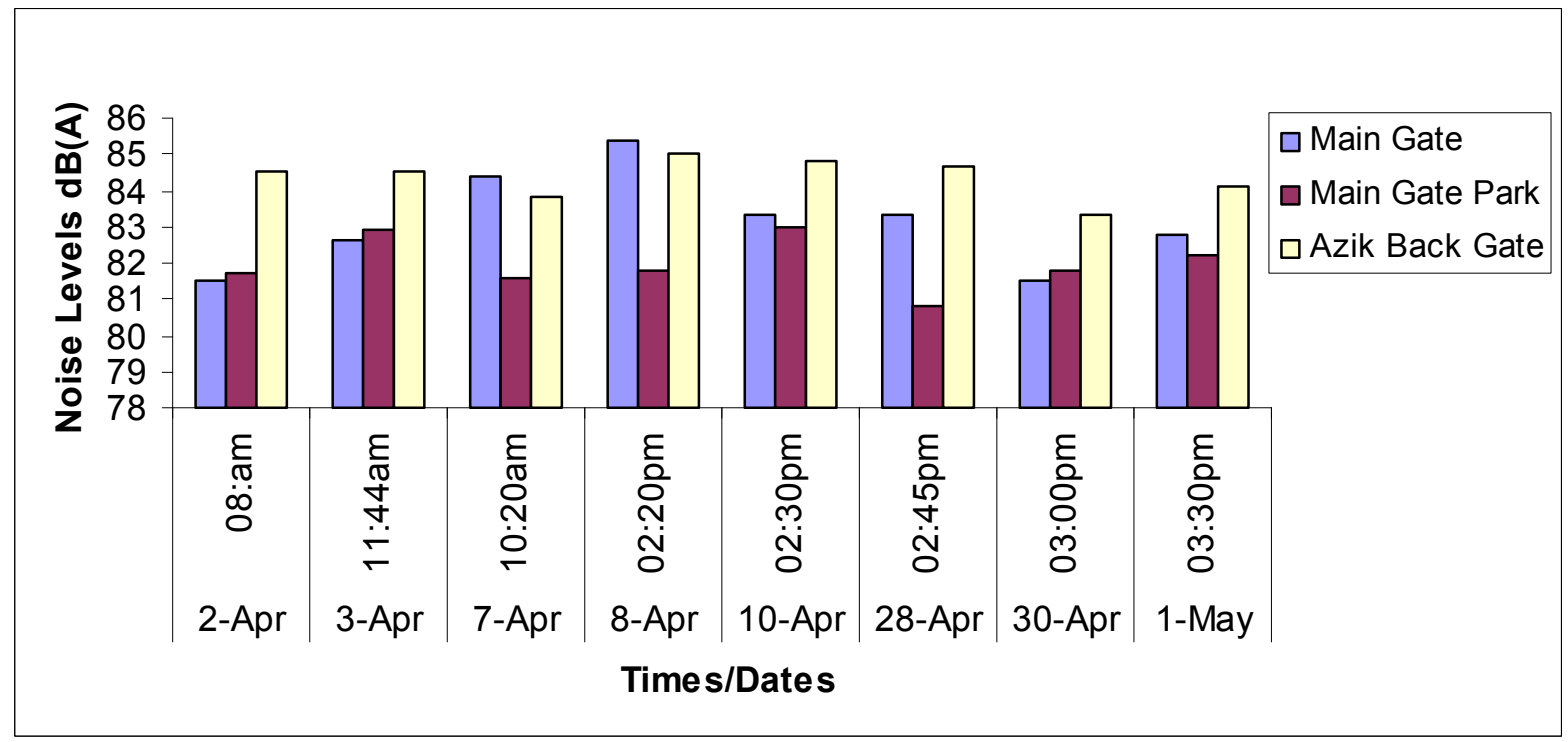

Fig. 3.1. Variations of Maximum Noise Levels with Time and Dates Around University Gates.

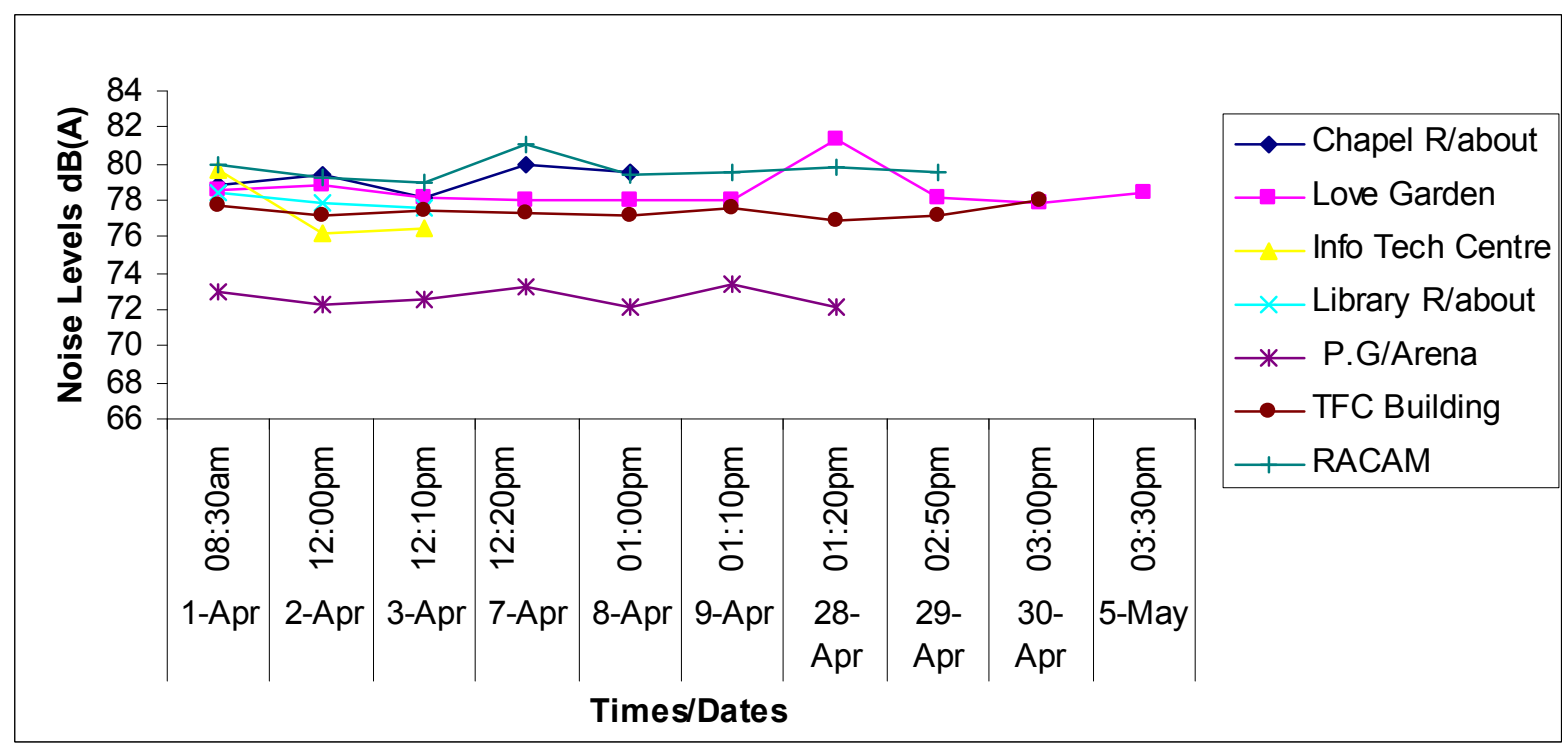

Fig. 3.2. Variations of Maximum Noise Levels with Time and Date along Major Road Junctions. 


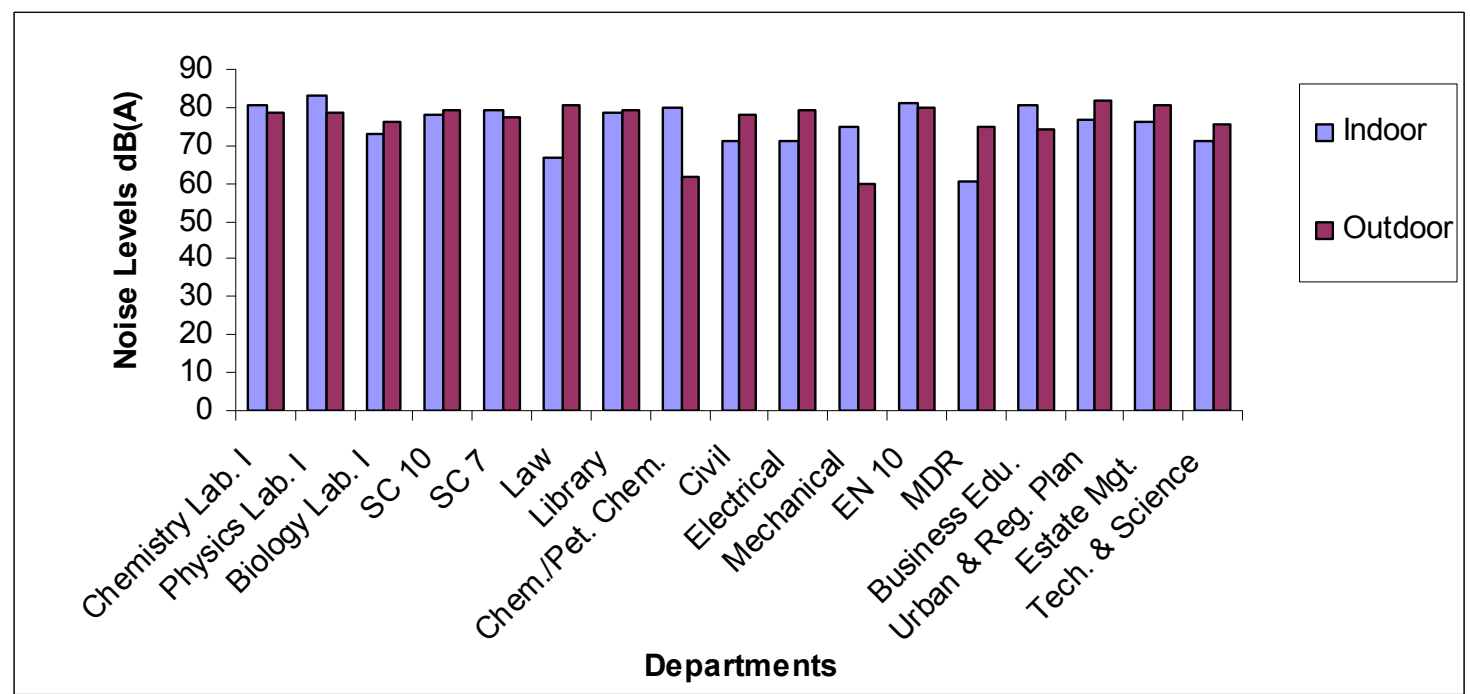

Fig. 3.3. Comparison Between Indoor and Outdoor Noise Levels at Various Departments.

Table 3.4 and Fig. 3.4 show the noise levels ranging from $68.2-81.4 \mathrm{~dB}(\mathrm{~A})$ at hostel $\mathrm{B}, 64.1-82.8 \mathrm{~dB}(\mathrm{~A})$ at Hostel C, $63.2-86.4 \mathrm{~dB}(\mathrm{~A})$ at Hostel $\mathrm{F}$ and $68.1-86.8 \mathrm{~dB}(\mathrm{~A})$ at Hostel G. In all, the results ranged from $62.7 \mathrm{~dB}$ (A) (between Hostels F \& G) to $86.8 \mathrm{~dB}$ (A) (Hostel F) with a mean maximum of $81.0 \mathrm{~dB}(\mathrm{~A})$ (Hostel $\mathrm{C}$ ).

Table 3.5 and Fig. 3.5 show the results of measurements at the electricity generating power plant houses. The ambient noise levels ranged from $98.2-103.2 \mathrm{~dB}$ (A) with a maximum mean of $100.4 \mathrm{~dB}(\mathrm{~A})$, at $50 \mathrm{~m}$ away, it ranged between $70.0 \mathrm{~dB}(\mathrm{~A})$ and $83.6 \mathrm{~dB}(\mathrm{~A})$ with a maximum mean of $76.8 \mathrm{~dB}(\mathrm{~A})$, at $100 \mathrm{~m}$ away it ranged from $58.5-78.4 \mathrm{~dB}$
(A) with a maximum mean of $68.6 \mathrm{~dB}(\mathrm{~A})$.

The results of noise levels obtained at International Secondary School varied from $54.5 \mathrm{~dB}(\mathrm{~A})-72.7 \mathrm{~dB}(\mathrm{~A})$ at the gate, $69.0 \mathrm{~dB}(\mathrm{~A})-80.1 \mathrm{~dB}(\mathrm{~A})$ at the senior block, 69.0 $\mathrm{dB}(\mathrm{A})-82.8 \mathrm{~dB}(\mathrm{~A})$ at the Junior block and $70.0 \mathrm{~dB}(\mathrm{~A})-$ $84.6 \mathrm{~dB}(\mathrm{~A})$ at the field with a maximum mean of $77.4 \mathrm{~dB}(\mathrm{~A})$ (Table 3.6 and Fig. 3.6).

The noise levels results measured at the Farm site and Security village ranged from 54.0 - $67.5 \mathrm{~dB}$ (A) at Power Outreach Ministry, 50.1 - $73.8 \mathrm{~dB}$ (A) at Deeper Life Church, 53.0 - $56.8 \mathrm{~dB}(\mathrm{~A})$ behind main gate car park and 54.2 - 71.2 $\mathrm{dB}(\mathrm{A})$ at the Farm site (Table 3.7 and Fig. 3.7).

Table 3.4. Noise Levels Measured at the Hostels.

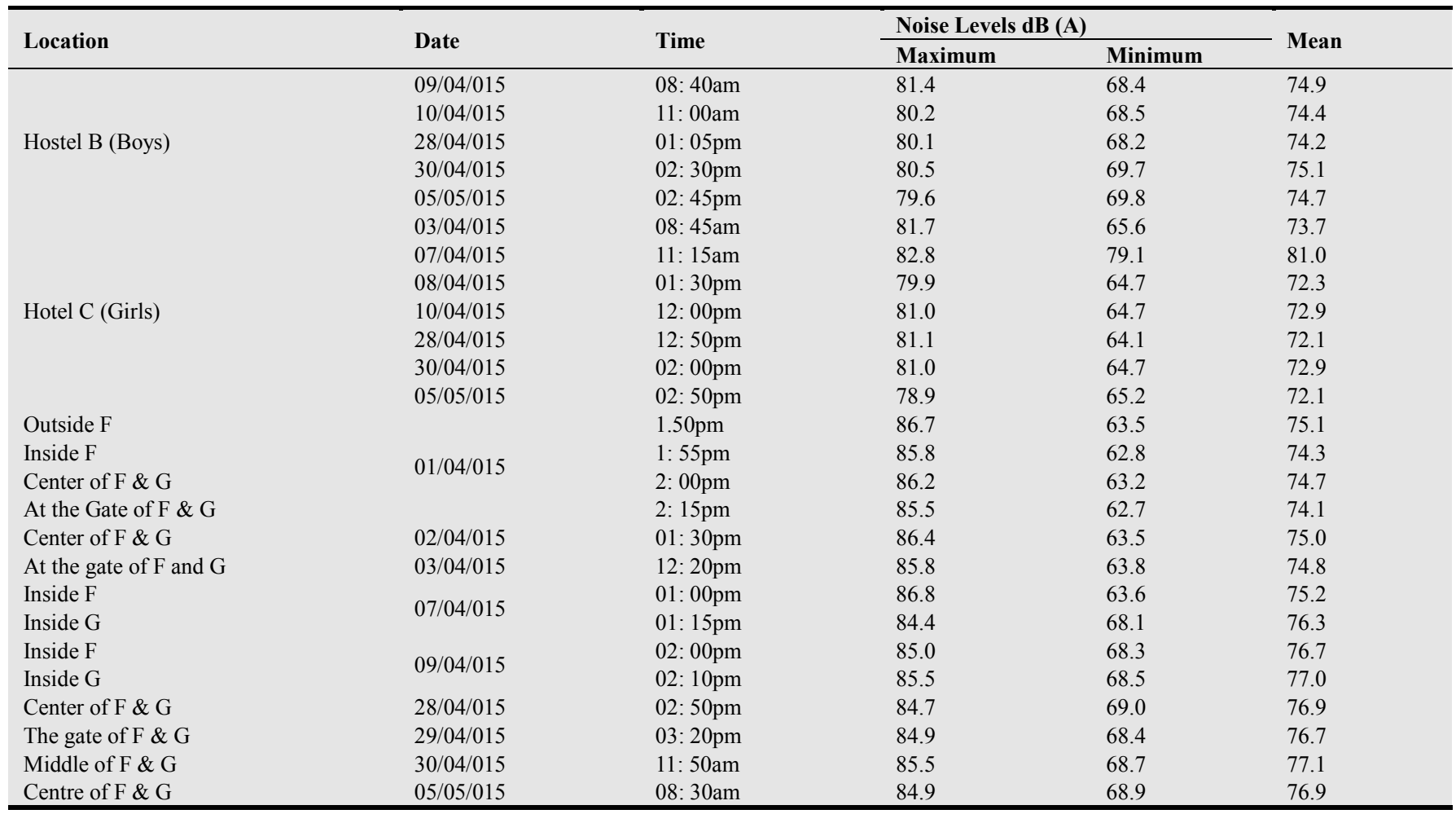




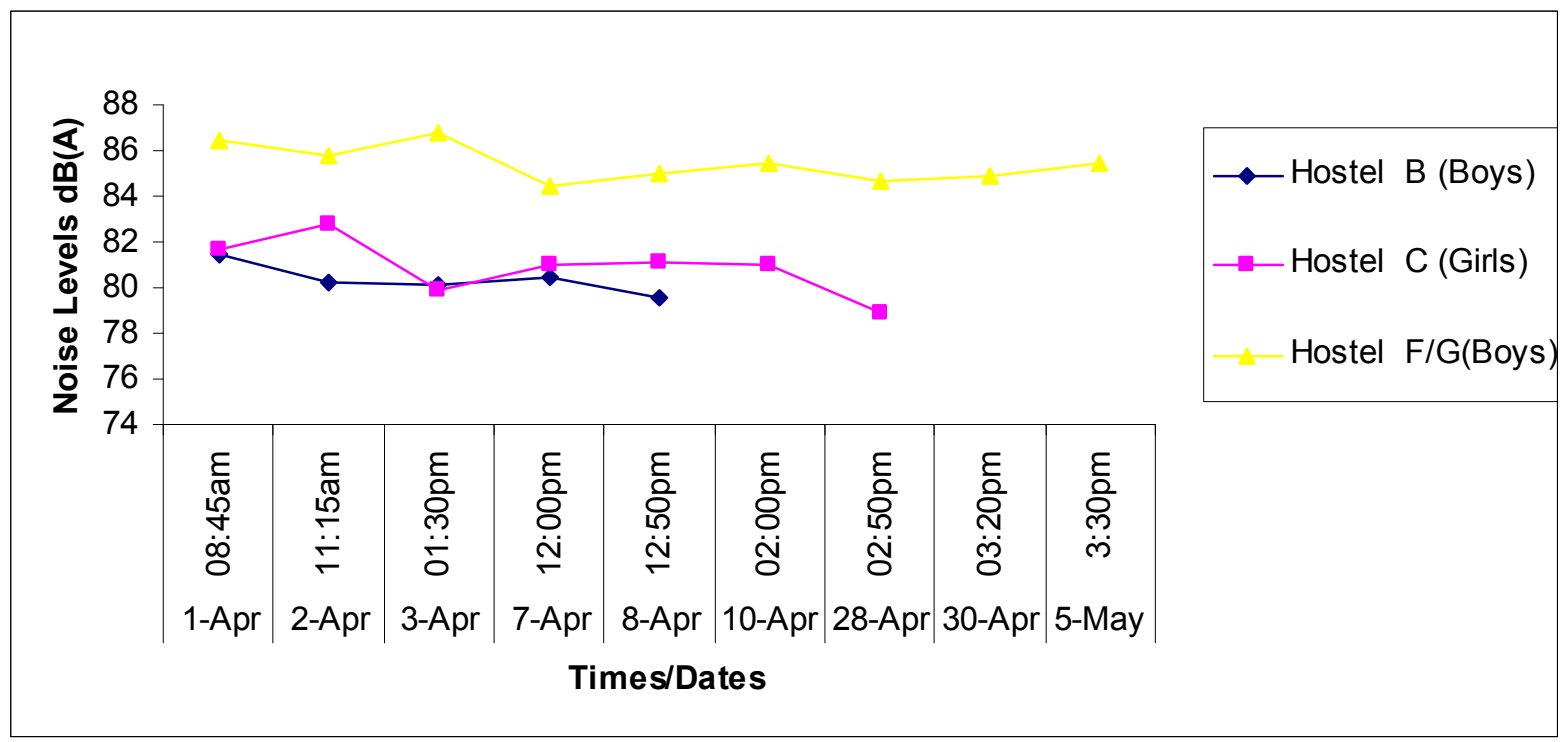

Fig. 3.4. Variations of Maximum Noise Levels with Time and Dates at Hostels.

Table 3.5. Ambient Noise Levels around University Electricity Generating Plant Houses.

\begin{tabular}{|c|c|c|c|c|c|c|c|}
\hline \multirow{3}{*}{ Location } & \multirow{3}{*}{ Date } & \multirow{3}{*}{ Time } & \multicolumn{4}{|c|}{ Noise Levels dB (A) } & \multirow{3}{*}{ Mean } \\
\hline & & & \multicolumn{2}{|c|}{ Maximum } & \multicolumn{2}{|c|}{ Minimum } & \\
\hline & & & Old site & New site & Old site & New site & \\
\hline \multirow{6}{*}{$0 \mathrm{~m}$} & $07 / 04 / 15$ & 08: 30am & 102.8 & 103.0 & 98.4 & 100.0 & 101.1 \\
\hline & $08 / 04 / 15$ & 09: 00am & 103.2 & 101.3 & 98.2 & 100.1 & 100.7 \\
\hline & $10 / 04 / 15$ & 09: 30am & 102.6 & 101.0 & 98.5 & 100.3 & 100.6 \\
\hline & $30 / 04 / 15$ & 11: 00am & 100.0 & 102.0 & 99.8 & 99.7 & 100.4 \\
\hline & $05 / 05 / 15$ & 11: $15 \mathrm{am}$ & 101.1 & 101.2 & 99.7 & 99.9 & 100.5 \\
\hline & $07 / 04 / 15$ & 11: 30am & 83.6 & 80.3 & 70.1 & 73.0 & 76.8 \\
\hline \multirow{3}{*}{$50 \mathrm{~m}$} & $08 / 04 / 15$ & 12: $00 \mathrm{pm}$ & 80.9 & 80.3 & 70.0 & 73.5 & 76.2 \\
\hline & $10 / 04 / 15$ & $12: 30 \mathrm{pm}$ & 81.1 & 80.0 & 70.5 & 732 & 76.2 \\
\hline & $05 / 05 / 15$ & $12: 50 \mathrm{pm}$ & 81.0 & 80.1 & 70.3 & 73.1 & 76.1 \\
\hline \multirow{4}{*}{$100 \mathrm{~m}$} & $07 / 04 / 15$ & $12: 30 \mathrm{pm}$ & 76.6 & 78.0 & 59.5 & 59.8 & 68.5 \\
\hline & $08 / 04 / 15$ & 01:00pm & 76.5 & 78.4 & 59.7 & 59.7 & 68.6 \\
\hline & $10 / 04 / 15$ & $01: 15 \mathrm{pm}$ & 76.2 & 78.2 & 59.2 & 58.7 & $68 . \mathrm{i}$ \\
\hline & $30 / 04 / 15$ & $01: 30 \mathrm{pm}$ & 76.0 & 78.2 & 59.5 & 58.5 & 68.1 \\
\hline
\end{tabular}

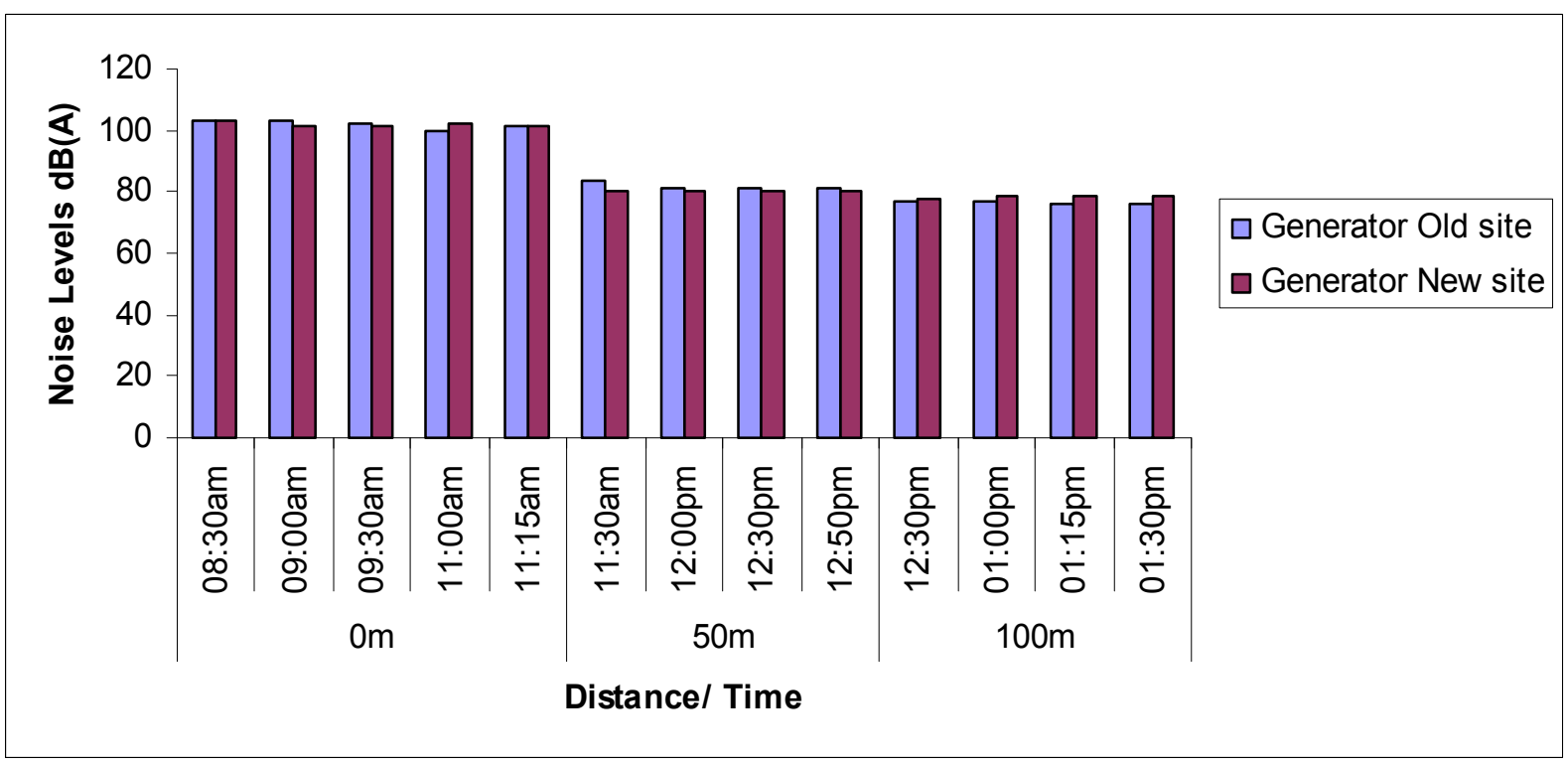

Fig. 3.5. Variations of Maximum Generator Noise Levels with Distance and Time. 
Table 3.6. Noise levels at International Secondary School (ISS).

\begin{tabular}{|c|c|c|c|c|c|}
\hline \multirow{2}{*}{ Location } & \multirow{2}{*}{ Date } & \multirow{2}{*}{ Time } & \multicolumn{3}{|c|}{ Noise Levels dB (A) } \\
\hline & & & Maximum & Minimum & Mean \\
\hline \multirow{7}{*}{ Gate } & \multirow{3}{*}{$06 / 05 / 015$} & 09: 35am & 71.8 & 54.6 & 63.2 \\
\hline & & $12: 05 \mathrm{pm}$ & 72.0 & 54.5 & 63.3 \\
\hline & & $02: 41 \mathrm{pm}$ & 71.9 & 54.8 & 63.4 \\
\hline & \multirow{3}{*}{ 07/05/015 } & 08: $26 \mathrm{am}$ & 72.7 & 54.7 & 63.7 \\
\hline & & $12: 10 \mathrm{pm}$ & 72.1 & 54.9 & 63.5 \\
\hline & & $02: 35 \mathrm{pm}$ & 72.3 & 54.8 & 63.6 \\
\hline & \multirow{3}{*}{$06 / 05 / 015$} & 09: 43am & 79.7 & 68.9 & 74.3 \\
\hline \multirow{5}{*}{ Senior block } & & $12: 10 \mathrm{pm}$ & 80.1 & 69.0 & 74.6 \\
\hline & & $02: 47 \mathrm{pm}$ & 79.4 & 69.3 & 74.4 \\
\hline & \multirow{3}{*}{$07 / 05 / 015$} & 08: 34am & 79.6 & 69.1 & 74.4 \\
\hline & & $12: 18 \mathrm{pm}$ & 79.8 & 69.1 & 74.5 \\
\hline & & $02: 42 \mathrm{pm}$ & 79.7 & 69.0 & 74.4 \\
\hline \multirow{6}{*}{ Junior block } & \multirow{3}{*}{$06 / 05 / 015$} & 09: 48am & 82.8 & 69.2 & 76.0 \\
\hline & & $12: 15 \mathrm{pm}$ & 82.1 & 69.8 & 76.0 \\
\hline & & $02: 52 \mathrm{pm}$ & 82.4 & 69.3 & 75.9 \\
\hline & \multirow{3}{*}{ 07/05/015 } & 08: 38am & 82.7 & 69.3 & 76.0 \\
\hline & & $12: 22 \mathrm{pm}$ & 82.5 & 69.5 & 76.0 \\
\hline & & $02: 47 \mathrm{pm}$ & 82.4 & 69.0 & 75.7 \\
\hline \multirow{6}{*}{ Field } & \multirow{3}{*}{$06 / 05 / 015$} & 09: $54 \mathrm{am}$ & 84.5 & 70.0 & 77.3 \\
\hline & & $12: 22 \mathrm{pm}$ & 84.1 & 70.2 & 77.2 \\
\hline & & 03: $00 \mathrm{pm}$ & 84.4 & 70.0 & 77.2 \\
\hline & \multirow{3}{*}{$07 / 05 / 015$} & 08: 45am & 84.1 & 70.1 & 77.1 \\
\hline & & $12: 27 \mathrm{pm}$ & 84.2 & 70.1 & 77.2 \\
\hline & & $02: 54 \mathrm{pm}$ & 84.6 & 70.2 & 77.4 \\
\hline
\end{tabular}

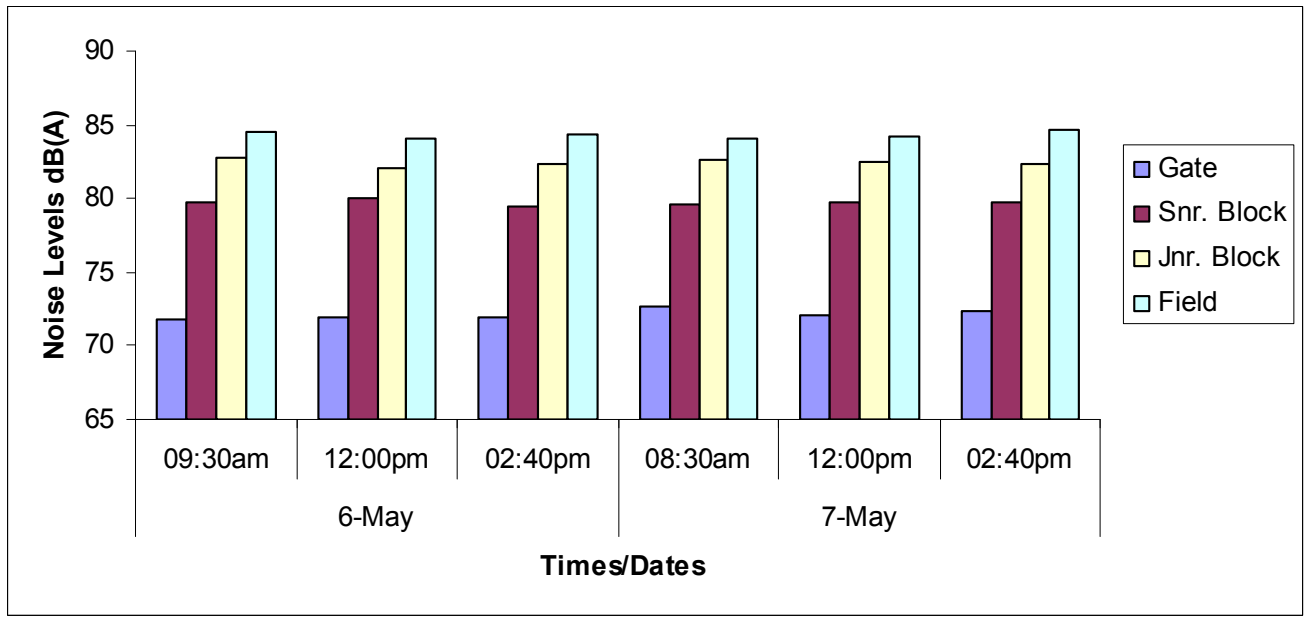

Fig. 3.6. Variations of Maximum Noise Levels with Time and Date at ISS.

Table 3.7. Noise Levels Measured at Farm Site and Security village in RSUST.

\begin{tabular}{|c|c|c|c|c|c|}
\hline \multirow{2}{*}{ Location } & \multirow{2}{*}{ Date } & \multirow{2}{*}{ Time } & \multicolumn{3}{|c|}{ Noise Levels dB (A) } \\
\hline & & & Maximum & Minimum & Mean \\
\hline \multirow{5}{*}{ Power outreach Ministry } & \multirow{2}{*}{$06 / 05 / 015$} & 10: 08am & 67.3 & 54.0 & 60.7 \\
\hline & & 03: $11 \mathrm{pm}$ & 67.1 & 54.2 & 60.8 \\
\hline & \multirow{3}{*}{$07 / 05 / 015$} & 08: $17 \mathrm{am}$ & 67.3 & 54.3 & 60.8 \\
\hline & & $12: 39 \mathrm{pm}$ & 67.2 & 54.1 & 60.7 \\
\hline & & $03: 10 \mathrm{pm}$ & 67.5 & 54.0 & 60.8 \\
\hline \multirow{4}{*}{ Deeper life Church } & $06 / 05 / 015$ & 10: $29 \mathrm{am}$ & 73.8 & 50.2 & 62.0 \\
\hline & \multirow{3}{*}{ 07/05/015 } & $08: 12 \mathrm{am}$ & 73.6 & 50.1 & 61.9 \\
\hline & & $12: 43 \mathrm{pm}$ & 73.3 & 50.4 & 61.9 \\
\hline & & $03: 15 \mathrm{pm}$ & 73.5 & 50.5 & 62.0 \\
\hline \multirow{4}{*}{ Behind Main Gate Park } & \multirow{2}{*}{$06 / 05 / 015$} & $10: 37 \mathrm{am}$ & 56.8 & 53.0 & 54.9 \\
\hline & & 03: $20 \mathrm{pm}$ & 56.6 & 53.5 & 55.1 \\
\hline & \multirow[b]{2}{*}{$07 / 05 / 015$} & 08: 06am & 56.3 & 53.2 & 54.8 \\
\hline & & 01: $01 \mathrm{pm}$ & 56.2 & 53.3 & 54.8 \\
\hline
\end{tabular}




\begin{tabular}{|c|c|c|c|c|c|}
\hline \multirow{2}{*}{ Location } & \multirow{2}{*}{ Date } & \multirow{2}{*}{ Time } & \multicolumn{3}{|c|}{ Noise Levels dB (A) } \\
\hline & & & Maximum & Minimum & Mean \\
\hline \multirow{5}{*}{ RSUST Farm Site } & $02 / 04 / 015$ & 08: 30am & 69.8 & 54.2 & 62.0 \\
\hline & $07 / 04 / 015$ & $12: 30 \mathrm{pm}$ & 70.4 & 54.7 & 62.6 \\
\hline & $10 / 04 / 015$ & 01: $00 \mathrm{pm}$ & 70.2 & 55.9 & 63.1 \\
\hline & $28 / 04 / 015$ & 03: $00 \mathrm{pm}$ & 71.2 & 54.6 & 62.9 \\
\hline & $01 / 05 / 015$ & $03: 50 \mathrm{pm}$ & 70.1 & 55.8 & 63.0 \\
\hline
\end{tabular}

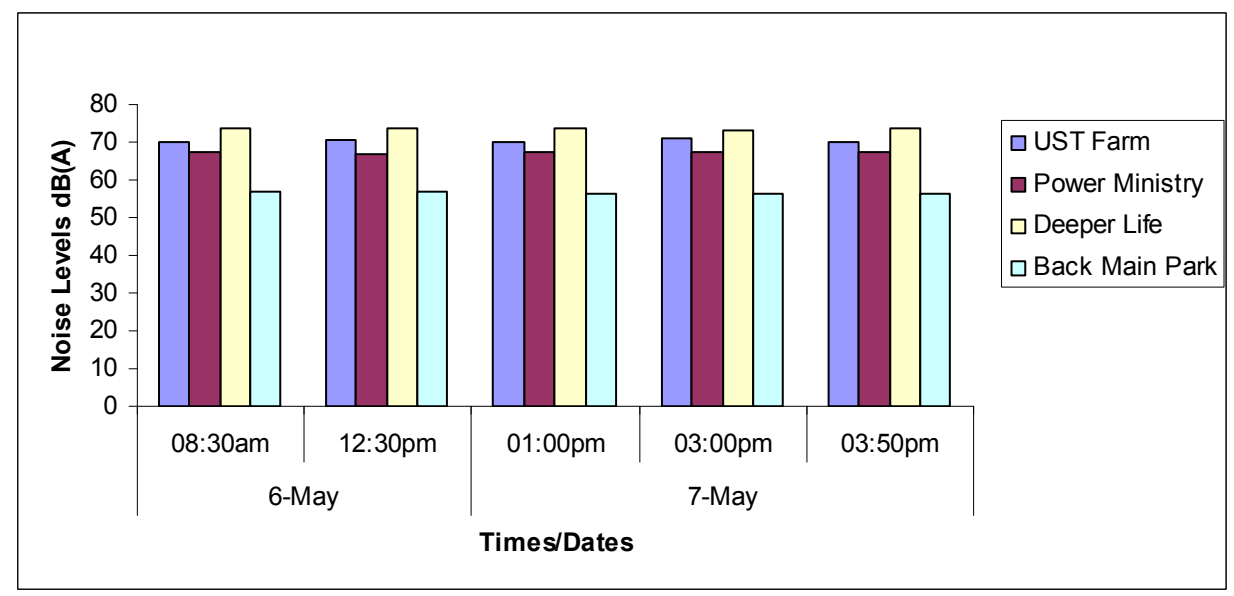

Fig. 3.7. Variations of Maximum Noise Levels with Time and Date at Farm/Security Village.

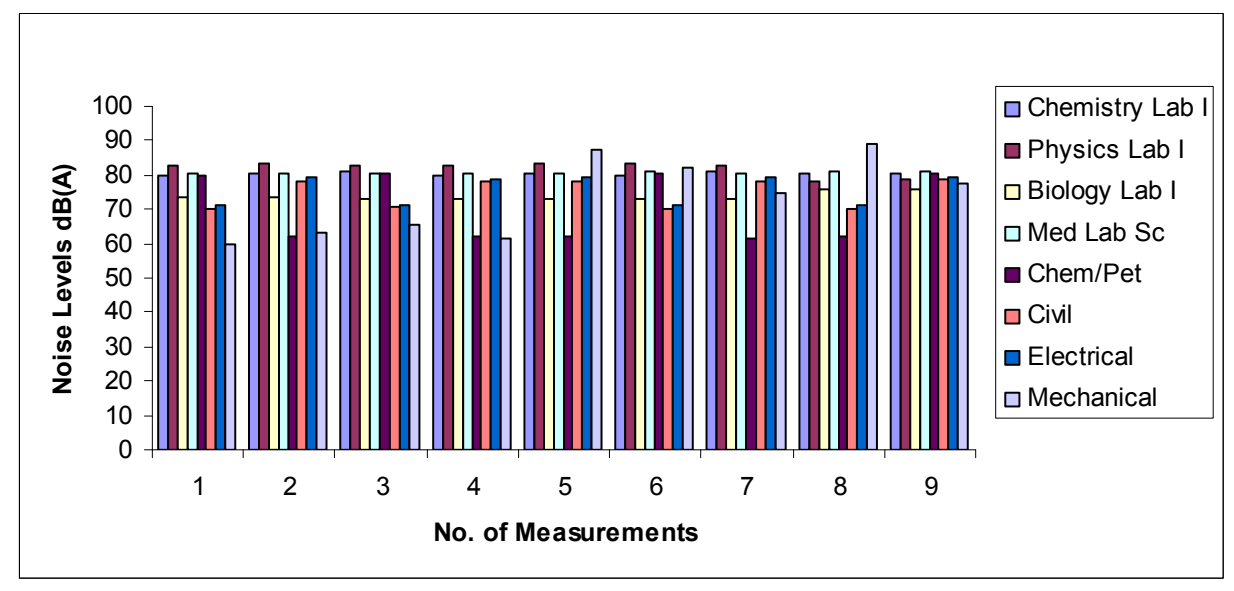

Fig. 3.8. Comparison of Maximum Noise Levels Between Departments.

\subsection{Discussion}

The noise levels at the main gate were found to be higher than that of Azikiwe gate and the main gate car park. This is due to vehicular activities and generators used by business centers outside the gate. The ANOVA between the noise levels at the gates and car park showed significant difference $(\mathrm{P}<0.05)$.

Along the major roads, Love Garden and RACAM were found to have high noise levels compared to other parts of the roads. This could be due to the various activities such as companies publicity and advertisements of products, vehicle traffic, landing helicopter, etc. along the major roads, the ANOVA showed significant difference $(\mathrm{P}<0.05)$.

At the students' hostels, the noise levels were found to be the highest at $\mathrm{F} \& \mathrm{G}$ followed by $\mathrm{C}$ while hostel $\mathrm{B}$ had the lowest noise levels. This observation was a result of students playing football at the field beside $F \& G$ and loud conversation/communications inside $\mathrm{F} \& \mathrm{G}$. Hostel $\mathrm{C}$ has higher noise level than $\mathrm{B}$ because of noise from the business center. At the hostels, though $\mathrm{t}-$ test between boys and girls hostels showed no significant difference $(\mathrm{P}<0.05)$ but ANOVA between the hostels showed significant difference $(\mathrm{P}<0.05)$.

The variations of noise levels at the generator houses indicate a reducing trend away from the generator. It was found that the noise levels at the new site were higher at six of the measurement times while the old site noise levels were also higher at six of the measurement times. The $t$ - test between the mean noise levels of the two generator sites showed no significant difference $(\mathrm{P}<0.5)$. There are two functional 2000KVA and $1275 \mathrm{KVA}$ generators at each site of the University. The difference in noise levels at the generator houses could be due to the generator capacity in operation at the time of measurement. The alternate use of the two generators could also have influenced the variations in noise 
levels.

At the International Secondary School, the nose level at the field were found to be highest followed by junior block. The noise levels at the field were high because in addition to wind movement, students were playing on the field during some measurement period. The higher noise levels at junior block could be as a result of uncontrolled conversations, shouts and play by the junior students. The ANOVA showed significant difference $(\mathrm{P}<0.5)$. The noise measured at the farm and security village showed that deeper life church site had high noise levels. This is due to church service with enhanced sound instrument. The noise levels at UST farm site were influenced by vehicles from Agip road and Agip helicopters. ANOVA showed significant difference between farm and security village noise levels.

Comparison of noise levels between Departments showed highest at mechanical workshop. This is due to heavy machines used in the workshop. ANOVA on inter faculties noise levels showed significant difference between faculties of Science and Engineering $(\mathrm{P}<0.5)$ while the intra department noise level in science with date showed significant difference $(\mathrm{P}<0.05)$. The trend of noise levels at the various Departments followed the order:

Mechanical $>$ Physics $>$ Urban and Regional $>$ Chemistry $>$ Medical Laboratory $>$ Estate Management $>$ Chemical/ Petrochem Eng. $>$ Electrical Eng. > Civil Eng. $>$ Law $>$ Biology $>$ Bus Education $>$ Technical and Science and the trend at lecture rooms followed the order: $\mathrm{EN} 10>\mathrm{SC} 10>\mathrm{SC}$ $7>\mathrm{NEH}>$ MDR.

The $\mathrm{t}$ - test between maximum and minimum noise levels at various departments showed significant difference $(\mathrm{P}<0.05)$ and positive correlation $(\mathrm{r}=0.5179)$.

Outdoor noise levels were generally higher than indoor levels, except at physics Department and SC7 which were influenced by the activity of gardens using Lawn mowers behind the physics laboratory during one of the measurement days. This observation is not in agreement with the report of Sonibare et. al. (2004). The indoor noise levels were higher at 7 locations while the outdoor noise levels were higher at 10 locations (Table 3.3). Generally, the outdoor levels were higher than indoor but $\mathrm{t}$ - test showed no significant difference $(\mathrm{P}>$ $0.5)$ between them.
The noise levels measured within the university were compared with national and international guideline values. The noise levels measured at the university main electricity generating plants exceeded both national and international guidelines.

However, the mean noise levels measured in all parts of the university exceeded the international guideline value recommended for a school (learning) environment.

WHO (1999) recommended guide line values of $35 \mathrm{~dB}(\mathrm{~A})$ for school class rooms (indoors) and $55 \mathrm{~dB}$ (A) (school outdoors) for community noise. London Times (1968) reported 85 dB (A), while Federal Ministry of Environment (FMEnv, 1991) Nigeria recommended 90dB (A) for an 8 hour working period.

Correlation matrices (Tables 3.9 and 3.10) show positive and negative correlation coefficients. The negative correlation coefficient between indoors and outdoors locations suggest little or no impact of activities outdoors on the indoor environment's noise levels in the university while the positive correlation coefficients indicate serious impact. High positive correlation coefficients between indoors and outdoors were recorded between physics indoor and EN10 outdoor ( $\mathrm{r}=$ 0.9820), physics outdoor and SC10 indoor $(\mathrm{r}=0.9891)$, physics outdoor and EN10 indoor $(\mathrm{r}=0.9078)$, physics outdoor and MDR indoor $(\mathrm{r}=0.9959), \mathrm{SC} 10$ indoor and EN10 outdoor $(r=0.9608)$, SC10 indoor and MDR indoor $(r=$ 0.9707), Civil Indoor and EN10 outdoor $(r=0.9820)$, Electrical outdoor and MDR outdoor $(r=0.9222)$, Mechanical outdoor and MDR indoor ( $\mathrm{r}=0.9222)$, EN10 outdoor and Law outdoor $(\mathrm{r}=0.9934)$. In comparing departments, high positive correlation coefficients were obtained between Chemistry Lab. and Urban \& Regional planning $(\mathrm{r}=0.9522)$, Civil and Electrical $(\mathrm{r}=0.9987)$.

Analysis of variance on noise levels between hostels, road junctions, gates and departments showed significant difference $(\mathrm{P}<0.05)$.

Noise levels outdoors were generally higher than indoors. $t$ tests on the mean noise levels between indoors and outdoors were not significant $(\mathrm{P}>0.05)$ but $\mathrm{t}$ - tests between maximum and minimum noise levels at the departments were significant $(\mathrm{P}<0.05)$.

Table 3.8. Analysis of Variance between sections of the University.

\begin{tabular}{|c|c|c|c|c|c|c|}
\hline \multicolumn{7}{|l|}{ ANOVA } \\
\hline Source of Variation & SS & df & MS & $\mathbf{F}$ & $P$-value & F crit \\
\hline Between Gates & 18.64095 & 2 & 9.320476 & 10.92853 & 0.000781 & 3.554557 \\
\hline Within Gates & 15.35143 & 18 & 0.852857 & & & \\
\hline Total & 33.99238 & 20 & & & & \\
\hline Between B, C, F/G & 65.14667 & 2 & 32.57333 & 36.82915 & $4.64 \mathrm{E}-05$ & 4.256495 \\
\hline Within B, C, F/G & 7.96 & 9 & 0.884444 & & & \\
\hline Total & 73.10667 & 11 & & & & \\
\hline Between Road Junctions & 128.425 & 4 & 32.10625 & 36.61614 & $1.42 \mathrm{E}-07$ & 3.055568 \\
\hline Within Road Juncs & 13.1525 & 15 & 0.876833 & & & \\
\hline Total & 141.5775 & 19 & & & & \\
\hline Between ISS Sections & 423.0855 & 3 & 141.0285 & 2161.356 & $4.49 \mathrm{E}-21$ & 3.238872 \\
\hline Within Sections & 1.044 & 16 & 0.06525 & & & \\
\hline Total & 424.1295 & 19 & & & & \\
\hline Between Farm/Security Village & 674.0419 & 3 & 224.6806 & 2636.839 & $3.54 \mathrm{E}-17$ & 3.490295 \\
\hline Within Groups & 1.0225 & 12 & 0.085208 & & & \\
\hline
\end{tabular}


Table 3.9. Correlation Matrix of Indoor and Outdoor Noise Levels.

\begin{tabular}{|c|c|c|c|c|c|c|c|c|c|c|c|c|c|}
\hline & Chem IN & $\begin{array}{l}\text { Chem } \\
\text { OUT }\end{array}$ & Phy IN & $\begin{array}{l}\text { Phy } \\
\text { OUT }\end{array}$ & Bio IN & Bio OUT & SC 7 IN & $\begin{array}{l}\text { SC } 7 \\
\text { OUT }\end{array}$ & SC 10 IN & $\begin{array}{l}\text { SC 10 } \\
\text { OUT }\end{array}$ & C/Pet IN & $\begin{array}{l}\text { C/Pet } \\
\text { OUT }\end{array}$ & Civil IN \\
\hline Chem IN & 1 & & & & & & & & & & & & \\
\hline Chem OUT & 0.54926 & 1 & & & & & & & & & & & \\
\hline Phy IN & 0.150095 & $\begin{array}{l}- \\
0.08112\end{array}$ & 1 & & & & & & & & & & \\
\hline Phy OUT & 0.40621 & 0.114468 & -0.20702 & 1 & & & & & & & & & \\
\hline Bio IN & -0.16583 & $\begin{array}{l}- \\
0.59315\end{array}$ & -0.11062 & $\begin{array}{l}- \\
0.17175\end{array}$ & 1 & & & & & & & & \\
\hline Bio OUT & -0.13214 & $\begin{array}{l}- \\
0.36212\end{array}$ & -0.71982 & 0.29036 & 0.534119 & 1 & & & & & & & \\
\hline SC 7 IN & -0.79373 & $\begin{array}{l}- \\
0.43231\end{array}$ & 0.31305 & $\begin{array}{l}- \\
0.69938\end{array}$ & $-7.5 E-15$ & -0.31068 & 1 & & & & & & \\
\hline SC 7 OUT & -0.13217 & $\begin{array}{l}- \\
0.86881\end{array}$ & 0.208514 & $\begin{array}{l}0.30434 \\
8\end{array}$ & 0.679608 & 0.472691 & 0.046625 & 1 & & & & & \\
\hline SC $10 \mathrm{IN}$ & 0.298807 & $\begin{array}{l}- \\
0.08839\end{array}$ & -0.22097 & 0.98909 & -0.07202 & 0.426597 & -0.63246 & 0.423896 & 1 & & & & \\
\hline SC 10 OUT & 0.761812 & 0.231125 & 0.27735 & -0.1253 & 0.401762 & -0.08113 & -0.49614 & 0.028916 & -0.1716 & 1 & & & \\
\hline C/Pet IN & 0.633058 & 0.652047 & -0.0532 & $\begin{array}{l}0.69751 \\
2\end{array}$ & -0.6821 & -0.24872 & -0.7151 & -0.4001 & 0.584178 & 0.059131 & 1 & & \\
\hline C/Pet OUT & 0.418121 & $\begin{array}{l}- \\
0.03164\end{array}$ & $-1.9 \mathrm{E}-15$ & $\begin{array}{l}0.54332 \\
4\end{array}$ & 0.38829 & 0.123448 & -0.89073 & 0.272096 & 0.509925 & 0.742891 & 0.412796 & 1 & \\
\hline Civil IN & -0.16176 & $\begin{array}{l}- \\
0.65043\end{array}$ & 0.101742 & $\begin{array}{l}0.53039 \\
5\end{array}$ & 0.214599 & 0.441201 & -0.3254 & 0.834441 & 0.836056 & -0.06727 & 0.085187 & $\begin{array}{l}0.29759 \\
5\end{array}$ & 1 \\
\hline Civil OUT & -0.34072 & -0.0532 & 0.595294 & $\begin{array}{l}- \\
0.21895\end{array}$ & -0.6027 & -0.79041 & 0.469776 & -0.397 & -0.25533 & -0.34597 & 0.213807 & -0.2786 & -0.0231 \\
\hline Elec IN & 0.88278 & 0.885615 & -0.13801 & $\begin{array}{l}0.41902 \\
6\end{array}$ & -0.43707 & -0.6455 & -0.91816 & -0.55205 & 0.276026 & 0.547723 & 0.828079 & $\begin{array}{l}0.65996 \\
6\end{array}$ & $\begin{array}{l}- \\
0.16667\end{array}$ \\
\hline Elec OUT & -0.33103 & 0.551246 & -0.34816 & $\begin{array}{l}- \\
0.50212\end{array}$ & -0.53585 & -0.30552 & 0.3114 & -0.88929 & -0.5693 & -0.33796 & 0.037113 & $\begin{array}{l}- \\
0.60974\end{array}$ & $\begin{array}{l}- \\
0.88662\end{array}$ \\
\hline Mech IN & \#DIV/0! & \#DIV/0! & \#DIV/0! & \#DIV/0! & \#DIV/0! & \#DIV/0! & \#DIV/0! & \#DIV/0! & \#DIV/0! & \#DIV/0! & \#DIV/0! & \#DIV/0! & \#DIV/0! \\
\hline Mech OUT & -0.00337 & 0.213293 & 0.740239 & $\begin{array}{l}- \\
0.37011\end{array}$ & -0.37152 & -0.45643 & 0.30669 & -0.2092 & -0.42356 & 0.277474 & 0.043748 & $\begin{array}{l}- \\
0.24724\end{array}$ & $\begin{array}{l}0.13909 \\
8\end{array}$ \\
\hline EN 10 IN & 1 & -1 & 1 & 1 & -1 & -1 & -1 & 1 & 1 & -1 & 1 & 1 & 1 \\
\hline EN 10 OUT & 0.211604 & $\begin{array}{l}- \\
0.17066\end{array}$ & 0.981981 & $\begin{array}{l}0.90784 \\
1\end{array}$ & -0.59604 & -0.32733 & -0.24019 & 0.654654 & 0.960769 & -0.5 & 0.5 & $\begin{array}{l}0.24019 \\
2\end{array}$ & $\begin{array}{l}0.98198 \\
1\end{array}$ \\
\hline MDR IN & 0.671932 & 0.344865 & 0.755929 & $\begin{array}{l}0.99587 \\
1\end{array}$ & -0.91766 & -0.75593 & -0.69338 & 0.188982 & 0.970725 & $4.11 \mathrm{E}-14$ & 0.866025 & $\begin{array}{l}0.69337 \\
5\end{array}$ & $\begin{array}{l}0.75592 \\
9\end{array}$ \\
\hline MDR OUT & -0.22069 & 0.487088 & -0.96609 & -0.7333 & -0.18732 & -0.2582 & 0.196748 & -0.82808 & -0.82808 & $1.3 \mathrm{E}-14$ & -0.13801 & $\begin{array}{l}- \\
0.37712\end{array}$ & -1 \\
\hline NEH IN & -0.66895 & $\begin{array}{l}- \\
0.84366\end{array}$ & 0.478091 & $\begin{array}{l}0.09072 \\
2\end{array}$ & 0.216295 & 0.447214 & 0.681554 & 0.717137 & 0.239046 & -0.63246 & -0.47809 & -0.4899 & 0.57735 \\
\hline NEH OUT & \#DIV/0! & \#DIV/0! & \#DIV/0! & \#DIV/0! & \#DIV/0! & \#DIV/0! & \#DIV/0! & \#DIV/0! & \#DIV/0! & \#DIV/0! & \#DIV/0! & \#DIV/0! & \#DIV/0! \\
\hline Law IN & 0.666204 & 0.788263 & 0.517298 & $\begin{array}{l}0.19518 \\
3\end{array}$ & -0.77165 & -0.80341 & -0.28642 & -0.50337 & 0.069673 & 0.218625 & 0.756262 & $\begin{array}{l}0.12857 \\
8\end{array}$ & $\begin{array}{l}- \\
0.14339\end{array}$ \\
\hline Law OUT & -0.07344 & 0.01786 & -0.04234 & $\begin{array}{l}0.71614 \\
7\end{array}$ & -0.17049 & -0.15229 & -0.1705 & 0.302082 & 0.835704 & -0.65558 & 0.400545 & $\begin{array}{l}0.20265 \\
5\end{array}$ & $\begin{array}{l}0.08213 \\
5\end{array}$ \\
\hline
\end{tabular}

Table 3.9. Continued.

\begin{tabular}{|c|c|c|c|c|c|c|c|c|c|c|c|c|c|}
\hline & Civil OUT & Elec IN & $\begin{array}{l}\text { Elec } \\
\text { OUT }\end{array}$ & Mech IN & $\begin{array}{l}\text { Mech } \\
\text { OUT }\end{array}$ & EN 10 IN & $\begin{array}{l}\text { EN 10 } \\
\text { OUT }\end{array}$ & MDR IN & $\begin{array}{l}\text { MDR } \\
\text { OUT }\end{array}$ & NEH IN & $\begin{array}{l}\text { NEH } \\
\text { OUT }\end{array}$ & Law IN & $\begin{array}{l}\text { Law } \\
\text { OUT }\end{array}$ \\
\hline Civil OUT & 1 & & & & & & & & & & & & \\
\hline Elec IN & $-3.4 \mathrm{E}-14$ & 1 & & & & & & & & & & & \\
\hline Elec OUT & 0.354291 & 0.113228 & 1 & & & & & & & & & & \\
\hline Mech IN & \#DIV/0! & \#DIV/0! & \#DIV/0! & 1 & & & & & & & & & \\
\hline Mech OUT & 0.604077 & 0.766238 & 0.029231 & \#DIV/0! & 1 & & & & & & & & \\
\hline EN 10 IN & 1 & \#DIV/0! & -1 & \#DIV/0! & 1 & 1 & & & & & & & \\
\hline EN 10 OUT & 1 & $4.1 \mathrm{E}-14$ & -0.98198 & \#DIV/0! & 0.605291 & 1 & 1 & & & & & & \\
\hline MDR IN & 0.866025 & 0.5 & -0.75593 & \#DIV/0! & 0.9222 & 1 & 0.866025 & 1 & & & & & \\
\hline MDR OUT & $-4.9 \mathrm{E}-15$ & 0.166667 & 0.905822 & \#DIV/0! & -0.5046 & -1 & -0.98198 & -0.75593 & 1 & & & & \\
\hline NEH IN & 0.239046 & -0.86603 & -0.39223 & \#DIV/0! & -0.37225 & 1 & 0.5 & 0 & -0.57735 & 1 & & & \\
\hline NEH OUT & \#DIV/0! & \#DIV/0! & \#DIV/0! & \#DIV/0! & \#DIV/0! & \#DIV/0! & \#DIV/0! & \#DIV/0! & \#DIV/0! & \#DIV/0! & 1 & & \\
\hline Law IN & 0.685714 & 0.868271 & 0.137219 & \#DIV/0! & 0.711033 & 1 & 0.427121 & 0.821995 & -0.07893 & -0.54687 & \#DIV/0! & 1 & \\
\hline Law OUT & 0.174754 & -0.1063 & -0.27874 & \#DIV/0! & -0.52961 & 1 & 0.993399 & 0.802955 & -0.63779 & 0.552345 & \#DIV/0! & 0.067618 & 1 \\
\hline
\end{tabular}


Table 3.10. Correlation Matrix of Noise Levels in the Departments.

\begin{tabular}{|c|c|c|c|c|c|c|c|c|c|}
\hline & Chemistry Lab I & Physics Lab I & Biology Lab I & Med Lab Sc & SC 7 & SC 10 & Chem/Pet & Civil & Electrical \\
\hline Chemistry Lab I & 1 & & & & & & & & \\
\hline Physics Lab I & -0.24412 & 1 & & & & & & & \\
\hline Biology Lab I & -0.04531 & -0.95164 & 1 & & & & & & \\
\hline Med Lab Sc & -0.44233 & -0.67073 & 0.616331 & 1 & & & & & \\
\hline $\mathrm{SC} 7$ & -0.30312 & 0.699682 & -0.66999 & -0.8089 & 1 & & & & \\
\hline SC 10 & 0.269084 & -0.66031 & 0.619372 & 0.849948 & -0.97261 & 1 & & & \\
\hline Chem/Pet & -0.21765 & -0.04888 & 0.178047 & 0.150473 & -0.10788 & -0.05625 & 1 & & \\
\hline Civil & -0.17423 & 0.113244 & -0.16207 & -0.33654 & 0.271906 & -0.22723 & -0.05307 & 1 & \\
\hline Electrical & 0.050535 & 0.144948 & -0.16371 & -0.33229 & 0.178855 & -0.11648 & -0.54217 & 0.998677 & 1 \\
\hline Mechanical & -0.00337 & -0.45406 & 0.427439 & 0.306647 & -0.56357 & 0.592454 & -0.16926 & -0.05921 & -0.04584 \\
\hline EN 10 & 0.817737 & 0.670694 & -0.36035 & -0.92672 & -0.31469 & -0.13217 & 0.053484 & -0.02292 & -0.03341 \\
\hline MDR & -0.90111 & -0.40962 & 0.323222 & 0.526033 & 0.152631 & -0.03446 & 0.16784 & -0.19283 & -0.1763 \\
\hline $\mathrm{NEH}$ & -0.66895 & 0.478091 & 0.216295 & 0.426401 & 0.681554 & 0.239046 & -0.71289 & 0.683927 & 0.719877 \\
\hline Law & -0.37465 & 0.085264 & 0.042563 & 0.006734 & 0.296408 & -0.19821 & 0.264153 & 0.014506 & -0.38179 \\
\hline Estate Mgt. & -0.82163 & 0.271448 & 0.192982 & 0.622543 & 0.82922 & 0.038778 & -0.69451 & 0.664206 & 0.696456 \\
\hline Bus. Edu. & 0.860073 & 0.239046 & -0.54074 & -0.8528 & -0.90874 & 0.717137 & 0.007749 & 0.027357 & 0 \\
\hline Urban \& Reg Plan & 0.952217 & 0.327327 & -0.9934 & -1 & -0.96077 & 0.720577 & 0.009464 & 0.033942 & 0 \\
\hline Tech. \& Sci & 1 & 1 & -1 & -1 & -1 & 1 & -1 & 1 & 1 \\
\hline
\end{tabular}

Table 3.10. Continued.

\begin{tabular}{|c|c|c|c|c|c|c|c|c|c|}
\hline & Mechanical & EN 10 & MDR & NEH & Law & Estate Mgt. & Bus. Edu. & Urban \& Reg Plan & Tech. \& Sci \\
\hline Mechanical & 1 & & & & & & & & \\
\hline EN 10 & 0.796709 & 1 & & & & & & & \\
\hline MDR & -0.44026 & -0.9906 & 1 & & & & & & \\
\hline $\mathrm{NEH}$ & -0.37225 & -0.74162 & 0.806696 & 1 & & & & & \\
\hline Law & 0.296043 & -0.04017 & 0.385353 & 0.658974 & 1 & & & & \\
\hline Estate Mgt. & -0.56449 & -0.87496 & 0.92075 & 0.973329 & 0.619024 & 1 & & & \\
\hline Bus. Edu. & 0.95491 & 0.87646 & -0.82411 & -0.5 & -0.63343 & -0.64889 & 1 & & \\
\hline Urban \& Reg Plan & 0.992005 & 0.928571 & -0.87512 & -0.5 & -0.84841 & -0.65465 & 1 & 1 & \\
\hline Tech. \& Sci & 1 & 1 & -1 & 1 & 1 & 1 & 1 & 1 & 1 \\
\hline
\end{tabular}

\section{Conclusion and Recommendations}

\subsection{Conclusion}

Based on the findings of the study, the noise levels measured in RSUST are said to be typical of urban communities. The status of noise in RSUST does not portend significant negative occupational noise impact, since the measured noise levels were generally below Occupational Safety Health Agency and Federal Ministry of Environment guidelines for 8 hours working day and exceeded WHO guidelines for school environment. Major sources of noise within RSUST were generators, automobiles, human conversations, machineries, helicopter and wind movement.

\subsection{Recommendation}

University Authority should provide steady power supply by Power Holding Company of Nigeria. Alternatively, solar plant or sound proof generators should be provided. Practical actions to limit and control the exposure to environmental noise are essential.

\section{Acknowledgement}

The author hereby sincerely acknowledges Confidence N. Oguamalam for the financial contribution and field measurements.

\section{References}

[1] Berger, E. H. (2000) "Noise Control and Hearing Conservation" why do it. In Berger, E. H. Royster, L. H., Royster, J. D., Driscoll, D. P., Layne, M (eds). 2000. The Noise Manual American Industrial Hygiene Association. 1 - 17.

[2] Bisio. G. (1996). Case history: noise level survey in middle size town and remarks on restrictions. Noise Control English Journal, 41, $201-206$.

[3] Demian, G., Demian, M., Grecu L. and Grelu, V. (2008). Studies about noise pollution in urban areas. Proceedings of the $10^{\text {th }}$ WSEAS International Conference on Acoustics, Music Theory and Applications of Bulglalria, 87 - 91.

[4] Goswami, S., Nayak, S. K., Pradham, A. C. and Dey, S. K. (2011). A study on traffic noise of two campuses of university, Balasore, India. Journal of Environ Biology, 32 (1), 105 - 109.

[5] Jefferson, C. (2013). Noise Pollution. U. S. Environmental Protection Agency. Retrieved 2013 - 09 - 24.

[6] Nelson, P. M. (1998). Transportation. Noise control English Journal, 46, 159 - 166.

[7] Niosh (1996) National Occupational Research Agenda. National Institute of Occupational Safety and Health, DHHS (NIOSH) Pub. No. 96 - 115. Cincinnati, OH.

[8] N. M. S. (1998). Nigerian Meteorological Services, pp. 1 - 10. 
[9] Onuu, M. U. (1992). Measurement and analysis of road traffic noise and its impact in part of South Eastern Nigeria. Ph. D thesis, University of Calabar, Calabar, Cross River State, Nigeria.

[10] Oyedepo, O. S. and Saadu, A. A. (2009). A comparative study of noise pollution levels in some selected areas in Ilorin metropolis, Nigeria. Environmental Monit Assessment, 158, $155-167$.

[11] Rosen, S. and Olin (1995). Hearing loss and coronary heart disease. Archives of Otolaryngology, 82, 236.

[12] RSUST, (2013). Rivers State University of Science and Technology, Port Harcourt, News Bulletin Vol. V. No, III \& IV December (2013). A quarterly publication of the information, Publication \& Protocol Unit, Office of the Vice Chancellor, Back Cover.

[13] Singh, N. and Daver, S. C. (2004). Noise pollution: Sources, effects and control. Journal of Human and Ecology, 16, 181 187.
[14] Sinha, S., and Sridharan, P. V. (1999); Present and Future Assessment of Noise Level in the Neyveli Region. Journal of Environmental Studies and Policy 2 (1), 1 - 13.

[15] Smoorenburg, G. F., Axelson, A., Babisch, W., Diamond, I. G., Isling, H., Marth, E., Miedeman, H. M. E, Ohistronm, E., Rice, C. G., Abbing, E. W. R., Van de Wiel, J. A. G., Passchier Vermeer, W. (2003). Effects of noise on Health. Noise News Int, 4 (4), $137-150$.

[16] Thakur, G. S. (2006). A study of noise around an educational institutional area. Journal of Environmental Science Engineering, 48 (1), 35 - 38.

[17] Ugwuanyi, J., Ahemen, U. I. and Agbendeh, A. A. (2005). Assessment of environmental noise pollution in Markurdi metropolis, Nigeria zuma. Journal of Pure Applied Science, 6, $134-138$.

[18] WHO, (2005). United Nations Road Safety Collaboration: A handbook of partner profiles. World Health Organization Geneva, Switzerland. 